\title{
Chemical and Physical Aspects of Self-Healing Materials
}

\author{
Ying Yang, Xiaochu Ding, and Marek W. Urban* \\ Department of Materials Science and Engineering \\ Center for Optical Materials Science and Engineering Technologies (COMSET) \\ Clemson University, Clemson, SC 29634
}

\begin{abstract}
The concept of self-healing synthetic materials emerged a couple of decades ago and continues to attract scientific community. Driven primarily by an opportunity to develop life-like materials on one hand, and sustainable technologies on the other, several successful approaches to repair mechanically damaged materials have been explored. This review examines chemical and physical processes occurring during self-healing of polymers as well as examines the role of interfaces in rigid nano-objects in multicomponent composites. The complex nature of processes involved in self-healing demands understanding of multi-level molecular and macroscopic events. Two aspects of self-healing are particularly intriguing: physical flow (macro) of matter at or near a wound and chemical re-bonding (molecular)of cleaved bonds. These events usually occur concurrently, and depending upon interplay between kinetics and thermodynamics of the processes involved, these transient relations as well as efficiency are critical in designing self-healing materials. This review examines covalent bonding and supramolecular chemistry in the context of molecular heterogeneities in repair processes. Interfacial regions in nanocomposites also facilitate an opportunity for supramolecular assemblies or covalent bonding which, if designed properly, are capable of self-repairs.
\end{abstract}

Key words: Self-healing, heterogeneity, reversible covalent bonds, supramolecular chemistry, nanocomposites

*Corresponding author:mareku@clemson.edu 


\section{Table of Contents}

1. Introduction

2. Chemistry of Self-Healing and Heterogeneities

3. Nanocomposites and the Role of Interfaces

4. Physical Aspects of Self-Healing

5. Conclusions and Insights

Acknowledgements

References 


\section{Introduction}

Living organisms are able to recover from injuries to resume active and reproductive functions. The ability to continue these metabolic processes determines the longevity of their existence. Synthetic materials are designed to be functional, but do not possess metabolic attributes. To prolong their lifespan, the concept of self-repairing has been explored and became the central topic of scientific interests and technological significance. Over the last decade, numerous successful approaches to repair mechanically damaged materials have been explored, and recent advances have been summarized in several review articles.[1-6]

There are two events during self-repairing that are particularly critical: (1) physical flow of molecular segments at or near a wounded area and (2) re-bonding of cleaved bonds after mechanical damage. These events may occur continuously, depending upon interplay between kinetics and thermodynamics during these events. Ideally, one can envision diffusion of chain ends, followed by their re-bonding. The complexity in monitoring these interrelated events comes from the fact that reactivities of chain ends may not favor re-bonding. For example, when reactive free radicals are quenched immediately by atmospheric oxygen $\left(\mathrm{O}_{2}\right)$, water $\left(\mathrm{H}_{2} \mathrm{O}\right)$, or other reactive groups, chemical re-bonding will not occur. However, if diffusion continues, segmental motion may still lead to inter-diffusion and repair. Consider these circumstances, repair will be determined by the kinetic energy of chains and entropic changes during the diffusion. As has been shown,[5] these intrinsic properties will have significant impact on entropic contributions to the Gibbs free energy during repair. In contrast, when reactive chain ends are stable, but segmental mobility is limited, repairs will not occur at all, unless there is external energy input.

A critical factor to achieve desirable mobility of polymer chains is the presence of free volume.[7-9] Without 'voids,' the repeating units and segments forming longer macromolecular chains will be irresponsive. Thus, the well-known concept of "free" volume ought to describe an average chain mobility typically reflected in the glass transition temperature $\left(\mathrm{T}_{\mathrm{g}}\right)$ that impact such properties as permeability, diffusivity, flexibility, and others.[10] Does this imply that all "soft", or low $\mathrm{T}_{\mathrm{g}}$, polymer networks 
are able to self-heal? If this was the case, all pressure sensitive adhesives (PSA) should offer self-healing properties. Experiments teach us that this is not the case. Thus, the question how to create "voids" that may facilitate segmental mobility and, at the same time, high $T_{g}$, remains open. One option is to create a polymer system that will be heterogeneous, which can be accomplished by either phase separation, or combining soft and hard segments into one network by utilizing copolymers that can phase separate or composite materials. Another alternative is to utilize shape memory polymers (SMP), which will facilitate restoration to original shape, thus aiding in self-repair.

Previous studies have shown that incorporating stimuli-responsive units into a copolymer backbone leads to endothermic stimuli-responsive transitions $\left(\mathrm{T}_{\mathrm{SR}}\right)$ which are above the $T_{g}$, and require free volume distributions achieved by copolymerizing stimuliresponsive units into non-responsive functional backbone.[10-12] Figure 1, A depicts a heterogeneous network that consists of spatially distributed soft and hard domains, which, upon mechanical damage, are able to rearrange, thus facilitating repair. This is manifested by segmental mobility of chains into the voids or free volume areas. The most successful example of using this concept was to incorporate soft polyamide segments capable of forming H-bonds into rigid polystyrene (PS) chains, forming a heterogeneous polymer consists of a spherical PS phase within a polyamide matrix.[13] Analogous to liquid crystalline polymers, these networks can rearrange which is facilitated by the localized $\mathrm{T}_{\mathrm{g}}$ variations, thus leading to spontaneous network rearrangements manifested by increased $\Delta \mathrm{S}$, and subsequent re-bonding. Excess free volume is also generated as a result of the void growth under external stress during mechanical damage,[14] which may facilitate chain rearrangements and repair.

In designing self-healing systems, it is critical to realize the length scale of heterogeneities. As depicted in Figure 1, B, at Angstrom ( $\AA$ ) levels, self-healing will occur by reforming bonds at sites, where chain cleavage occurred. Block, branched, or star polymers forming $\mathrm{nm}$-to- $\mu \mathrm{m}$ scale microphase separation will facilitate self-healing, and microcapsules or inorganic particles embedded in polymers will respond to larger wounds.[15] In essence, to rationally design self-repairing materials, it is important to (1) understand which chemical entities are capable of cleavage due to mechanical damage; (2) design networks to achieve localized segmental mobility upon mechanical damage; and 
(3) synchronize re-bonding dynamics with physical rearrangements of macromolecular reactive segments. These pre-requisites combined with network heterogeneities are typically a starting point in molecular design of networks that are responsive to macroscopic damages. The following sections outline the role of bond dynamics in the context of localized heterogeneities and network components that facilitate self-repair.

\section{Figure 1.}

\section{Chemistry of Self-Healing and Heterogeneities}

Due to limited chain mobility and a lack of bond reformation abilities, common polymers do not show self-repair attributes. The last decade resulted in the development of a few classes of reactions to offer self-repairing through the cleavage and reformation of specific bonds. These entities are incorporated into polymer networks, and are typically categorized into reversible covalent and non-covalent bonds. The presence of these reversible bonds introduces molecular level heterogeneities along a polymer backbone, or as pendent groups, which may further facilitate the formation of new phases with extended length scales. Contributions of dynamic bonds and network heterogeneities to self-healing will be discussed in the following sections in the context of reversible covalent bonds, supramolecular chemistry, and self-healing nanocomposites.

\subsection{Reversible Covalent Bonds}

Dissociation and association rates of the dynamic bonds as well as chain mobility are significant factors in designing self-repairing materials. Reversible covalent bonds are good candidates for developing self-repairing polymers due to their high bonding strength, which enhance the mechanical toughness of materials. Four types of selfrepairing covalent bonds have been utilized: (1) reversible cycloaddition reactions, (2) exchange reactions, (3) stable free radical mediated reshuffling reactions, and (4) heterocyclic compounds/carbohydrates facilitated bond reformations. One drawback of covalent self-repairing bonds is the high activation energy. For example, for polymers utilizing Diels-Alder (DA) reactions, where activation temperature of the retro-DA reaction responsible for the self-healing is $120^{\circ} \mathrm{C},[16]$ self-healing only occurs by 
heating the damaged material above this temperature. The advantage is that $T_{g}$ of the polymer can be as high as $100^{\circ} \mathrm{C}$, but high temperature exposure may not be desirable. Ideally, bond dissociation and reformation should proceed at ambient conditions. Since constrained network mobility becomes the limiting factor, the following section will address how this limitation can be overcome by incorporating heterogeneities, leading to the development of tough materials containing reversible covalent bonds that can repair at ambient conditions.

\subsubsection{Reversible Cycloaddition Reactions}

Due to their adaptability to various polymers, Diels-Alder and similar cycloaddition reactions have been extensively investigated. Adduct (exo or endo) formation via DA reaction between furan (diene) and malemide (dienophile) entities can be utilized to build the crosslinked polymer network.[16, 17] Retro-DA reactions, typically induced by elevated temperature and/or crack, result in the disconnection of diene and dienophile, and lower temperatures reconstruct the covalent bonds to repair damages. DA pairs have been incorporated in polyesters, epoxies, and polyamides,[1821] leading to the formation of self-healing networks with various properties. This is summarized in Table 1, A. As shown in Table 1, A (a) and (b), the $T_{g}$ can be tuned as high as $100{ }^{\circ} \mathrm{C}$, but the activation temperature of the retro-DA reaction is above the $\mathrm{T}_{\mathrm{g}}$, which requires heating to high temperatures to activate the self-repair process. Room temperature repairs of polyester containing DA pairs have also been observed (Table 1, A-c). However, whether DA linkages can dissociate under these conditions is not obvious. The self-healing may be attributed to the material's low $\mathrm{T}_{\mathrm{g}}$ or possibly other mechanism. Table 1, A (d) illustrated a thermally reversible rigid polyamide network whose $T_{g}$ is higher than the activation temperature of retro-DA reactions. Self-healing at temperatures $\sim 100{ }^{\circ} \mathrm{C}$ below the $\mathrm{T}_{\mathrm{g}}$ resulted in partial repair of the scratch. When chain diffusion is not a limiting factor above the $\mathrm{T}_{\mathrm{g}}$, designed heterogeneity using PEO-b-PFGE block copolymer, as shown in Table 1, A (e), did not significantly alter the mechanical properties or self-repairing efficiency compared with the polymer shown in Table 1, A (a).[21] Self-healing of high $T_{g}$ materials under ambient conditions are advantageous, but also challenging. DA pairs that are reversible within mild temperature range are rare and 
require elaborate synthesis, which compromise the practicality. A novel DA system consisting of cyanodithioester (CDTE) compound and cyclopentadiene (Cp), as shown in Table 1, B, was developed to overcome the limitation of slow self-healing kinetics of the previous systems. [22] Crosslinked network formed between CDTE based tetracrosslinker and linear poly(iBoA-nBA) end capped with $\mathrm{Cp}$ groups offer rapid reversiblility and self-healing between $70-120{ }^{\circ} \mathrm{C}$ without any catalyst.

In order for materials to self-repair without excessive heating, photochemical cycloadditions provide an optically induced self-healing process. These include $[2+2]$ cycloaddition of 1,1,1-tris-(cinnamoyloxy-methyl)ethane (TCE) (Table 1, C) [23] or coumarin (Table 1, D),[24] and [4+4] cycloaddition of anthracene derivatives (Table 1, E) [25]. Similar to DA reactions, cycloaddition reactions constructing polymer networks, leading to self-repair via four-member ring opening and closure modulated by exposure to light at different wavelengths. Specifically, polymerization of 1,1,1-tris(cinnamoyloxymethyl)ethane (TCE) monomers via photo-stimulated [2+2] cycloaddtion gives a rigid polymer.[23] After damage-repair cycle, approximately 6\% of the original Young's modulus can be recovered by photo radiation to reconstruct the cyclobutane crosslinks (Table 1, C). Since fragmentations occur at room temperature, self-repair is likely limited by segmental mobility.

The rational design of self-repairable heterogeneous networks should facilitate enhanced segmental mobility. When dihydroxyl coumarin derivatives are reacted with isophorone diisocyanate (IPDI) and polyethylene glycol (PEG) forming linear polyurethane chain, crosslinking occur upon dimerization of coumarin derivatives induced by $350 \mathrm{~nm}$ UV radiation, forming optical stimuli-responsive polyurethane (IDHPEG).[24] The reversibility of cycloaddition reactions is shown in Table 1, D. The detection of two $\mathrm{T}_{\mathrm{g}} \mathrm{s}$ upon crosslinking indicates the coexistence of a rigid phase consisting of coumarin dimmer and a mobile phase of PEG-polyurethane segments. Selfrepair conditions along with the \% recovery for cycloaddition reactions are summarized in Table 1. When PEG with lower molecular weight was used, only one $T_{g}$ is observed owning to good miscibility between soft and hard segments, but \% recovery can only reach to $16.2 \%$. This indicates that formation of rubbery domains resulting from microphase separation is critical for the reversible photoreactions. Another example is the 
use of dendritic building block containing anthracene groups on the periphery. Photocrosslinking through dimerization of anthracene groups increases the $T_{g}$ from $-46{ }^{\circ} \mathrm{C}$ for the dendritic macromonomer to $75{ }^{\circ} \mathrm{C}$ for crosslinked films (Table 1, E). Upon damage, photo-radiation at $254 \mathrm{~nm}$ regenerates the building block, allowing reorganization of macromolecular segments, followed by crosslinking upon exposure to $366 \mathrm{~nm}$ radiation.[25] The network rearrangements and reformations occur at room temperature, as shown in Figure 2. The recent study also reported that coumarin groups alone are able to facilitate self-healing. When coumarin pendant groups were grafted on to silicone chains, coumarin-coumarin self-associations through $\pi$-stacking result in physical crosslinking, and thermoactivated dissociation followed by re-association provide selfhealing ability.[26]

Table 1.

\section{Figure 2..}

\subsubsection{Exchange Reactions}

Dynamic exchange reactions between reversible covalent bonds that proceed under mild conditions offer another opportunity for the development of self-healing polymers. Different from thermal reversible DA reactions, the activation temperatures of these exchange reactions are lower. Figure 3, A illustrates dynamics of acylhydrazone, in which acylhydrazone formation from acylhydrazine and aldehyde groups is reversible in the presence of acid catalyst.[27] Incorporation of acylhydrazone into polyethylene oxide (PEO) networks resulting in a polymer gel that self-heal at room temperature without stimuli.[28] Despite its ability of interchanging bonds even at room temperature, limited studies have been conducted. And another challenge is to achieve quick exchange in solid state without solvent.

Disulfide bonds can undergo metathesis exchange reactions in which two neighboring S-S bonds are disrupted and reformed through free radical or ionic intermediates. [29, 30] The cleavage can be activated by photolysis, heating, or

oxidation.[31] Taking advantage of these conditions, disulfide interchange illustrated in 
Figure 3, B was utilized in self-repairing because S-S entities offer good compatibility with polymeric networks and room-temperature bond reversibility.[32, 33] Mechanical properties and self-repairing abilities vary, depending upon networks morphologies. This is summarized in Table 2, A though D. Table 2, A shows an example, in which S-S bonds were introduced into epoxy resin to obtain self-repair, resulting in a crosslinked homogeneous networks with the $\mathrm{T}_{\mathrm{g}}$ of approximately $-35^{\circ} \mathrm{C}$. No solvent is used in this process, and the tensile stress measurement showed almost complete recovery of properties after heating at $60^{\circ} \mathrm{C}$ for $1 \mathrm{~h}$.[32]

When star polymers grafted with poly(n-butyl acrylate) on crosslinked poly(ethylene glycol diacrylate) core were combined with S-S bonds, heterogeneous selfrepairable networks can be produced.[33] The presence of the star polymer shown in Figure 4, A facilitates formation of a network with the high mobility and low intrinsic viscosity, enabling high accessibility of functional groups at ambient healing temperatures. The corresponding properties are shown in Table 2, B. Significant recovery upon repair is achieved, but at the expense of mechanical integrity. To enhance mechanical properties, rigid aromatic segments were incorporated into epoxy matrices[34]. When aromatic segments are placed along an epoxy backbone, the $\mathrm{T}_{\mathrm{g}}$ increase to $-3.6{ }^{\circ} \mathrm{C}$ was observed, whereas the $\mathrm{T}_{\mathrm{g}}$ of fully aliphatic network is $-46{ }^{\circ} \mathrm{C}$. However, longer repair times and lower healing efficiencies were detected (Table 2, C). Figure 4, B depicts another heterogeneous inorganic-organic hybrid structure composed of silicone alkoxides inorganic phase and epoxy resin phase containing reversible tetrasulfide bonds.[35] A comparison of the mechanical and self-repair properties of the inorganic-organic hybrid (Table 2, D) with the aromatic segments enhanced S-S based epoxy networks (Table 2, C) indicates that incorporation of inorganic phase leads to the formation of a tougher material with higher self-repair efficiency. Thus, enhanced mechanical properties without compromising self-repairing efficiency by introducing heterogeneous phase were achieved.

Exchange reactions in silicone-based materials also facilitated self-repair. Known for their ability to restructure under certain conditions, crosslinked silicone based polymers containing ethylene bridges and active silanolate end groups synthesized from anionic ring-opening copolymerization of octamethylcyclotetrasiloxane $\left(\mathrm{D}_{4}\right)$ and 
bis(heptamethylcyclotetrasiloxanyl)-ethane (bis- $\mathrm{D}_{4}$ ) showed remodeling attributes.[36] Figure 3, C depicts the siloxane exchange reactions responsible for the "livingness" promoted by the presence of tetramethylammonium dimethylsilanolate anionic end groups, which maintain their activity under ambient conditions, thus providing "ready-torespond" active groups. A self-healing polyurea is developed by modifying urea nitrogen with bulky groups, as shown in Figure 3, D. The modification lowered the dissociation energy of urea $\mathrm{C}-\mathrm{N}$ bond, resulting in dynamic equilibrium between urea and amine ($\mathrm{NH}_{2}$ )/isocyanate (-NCO) reactive groups. When the hindered urea bonds (HUBs) are used to obtain polyurethane-ureas with sub-ambient glass transition temperature, selfrepairing under ambient conditions is achieved.[37] When utilizing free-NCO groups, even physically entrapped, one needs to realize their toxicity and reactivity toward $\mathrm{H}_{2} \mathrm{O}$.

\section{Figure 3.}

Table 2.

Figure 4.

\subsubsection{Stable Free Radical-Mediated Reshuffle Reactions}

As pointed out above, it is critical to provide sufficient time for covalent bonds to reform during network remodeling. Mechanical damage of polymers usually generates free radicals as a result of covalent bond cleavages. Recoupling of free radicals from opposite damaged surfaces will reconstruct the network by reforming covalent linkages. If the free radicals are not stabilized, oxidation processes will cause their reactivity loss, thus terminate self-healing. Dynamic reshuffling reactions involving stable free radical formation shown in Figure 5 offers an interesting alternative. Taking advantage of dynamic reshuffling of trithiocarbonates (TTC) via free radical mechanism shown in Figure 5, A, [38] where hemolysis of C-S bonds of TTC can be photostimulated by UV radiation at $330 \mathrm{~nm}$ generating stable free radicals which can exchange with neighboring TTC groups, self-repair property is attained. Thiuram disulfide moieties having similar reshuffling characteristics is also utilized to repair covalently cross-linked polymers.[39] Homolytic cleavage of the disulfide is triggered by visible light, resulting in formation of dithiocarbamate-based free radicals as shown in Figure 5, B. It was reported that such 
radicals are able to remain stable for more than two weeks,[40] thus providing sufficient time for network rearrangement before recoupling. TTC free radicals are sensitive to air, it is therefore desirable to seek free radicals that are air tolerant. For example, cleavage of diarylbibenzofuranone (DABBF) groups create arylbenzofuranone (ABF) oxygen insensitive radicals (Figure 5, C), thus making poly(propylene glycol) (PPG) polymer gels crosslinked by DABBF self-repairable.[41]

When polystyrene (PS) chains were crosslinked by dynamically reversible alkoxyamine (C-ON) groups, self-healing was achieved by thermal activated dissociation/association of alkoxyamine linkages, such as shown in Figure 5, D.[42] Crosslinking via C-ON groups makes already rigid PS even stiffer. Thus, the challenge is to gain sufficient mobility at the temperatures near the $T_{\mathrm{g}}$. As shown in Table 3, when molar ratio of styrene over the crosslinker $\mathrm{C}-\mathrm{ON}$ is $7.5 / 1$, two $\mathrm{T}_{\mathrm{g}} \mathrm{s}$ are detected. While the higher $\mathrm{T}_{\mathrm{g}}$ corresponds to crosslinked PS, the lower one is close to linear PS, indicating the existence of a mobile free PS phase arising from dynamic dissociations/associations of C-ON bond within the network. The heterogeneous phase cannot be detected when PS chains between crosslinking points are either shorter (styrene $/ \mathrm{N}-\mathrm{CO}=5 / 1$ ) or longer (styrene/ $\mathrm{N}-\mathrm{CO}=10 / 1)$. As expected, the network containing a mobile phase (styrene/N$\mathrm{CO}=7.5 / 1)$ offers the highest healing efficiency. When molar ratio of styrene over C-NO is $5 / 1$, free chains generated from scission of $\mathrm{N}-\mathrm{CO}$ are too short to form a new phase. Thus, self-healing is limited by restricted motion of macromolecular segments. When the ratio is $10 / 1$, the cleaved chains are long, and the chain flexibility will favor reassociation, and the healing efficiency is limited by lower concentrations of free radicals. This study demonstrated that the coexistence of a mobile phase within a rigid network is favorable for self-repairs. Activation temperature of alkoxyamines can be adjusted by placing them in different polymers,[43] and room temperature repair is obtained using linear polyurethane as the matrix[44]. The soft domains originate from PEG segments within polyurethane network facilitate the repair. One limitation of alkoxyamines is that their stability toward air is not clear. The oxidative processes may intercept re-bonding reactions.

\section{Figure 5.}

\section{Table 3}




\subsubsection{Heterocyclic Compounds and Carbohydrates in Self-Healing Polyurethanes}

When mechanical forces are evenly distributed within the network, bond rupture typically occurs at positions of lowest bond strength. For polymers, however, external forces are in fact unevenly distributed due to the presence of entanglements and crosslinked points. As a result, not only the weakest bonds, but also other covalent and non-covalent bonds may cleave depending upon the magnitude of the force.[45] While many of the self-healing studies focused only on one type of bond reformation, reactions that reconstruct multiple cleaved bonds are desirable. For example, chain cleavages resulting from mechanical damages of crosslinked polyurethanes will generate $-\mathrm{C} \cdot,-\mathrm{N}$ • and oxygen $-\mathrm{O} \cdot$ free radical intermediates. If free radicals remain stable until the chain ends come to close proximity and then recouple, self-repair may occur. For that reason, four and five member heterocyclic compounds, such as oxetanes (OXE) and oxolanes $(\mathrm{OXO})$, which offer low ring opening activation energy producing stable free radicals were utilized in developing self-repairable polyurethane (PUR) networks.[46, 47] The network is shown in Figure 6, A, where chitosan (CHI) was modified on the C6 position with the OXE, followed by crosslinking with tri-functional hexamethylene diisocyanate (HDI) in the presence of polyethylene glycol (PEG), forming a heterogeneous crosslinked PUR network (OXE-CHI-PUR). Upon mechanical damage, self-repair occurs by exposure to UV radiation. Specifically, as shown in Figure 6, A, four labile linkages are affected upon mechanically damage and UV radiation: NHCONH- PUA linkage, -C-O-C- glycosine units of CHI, -C-O-C- between CHI and $\mathrm{OXE}$, and -C-O-C- of OXE ring. Cleavages of these bonds generate free radicals and a carbocation. In addition, CHI pyranose ring absorb energy from UV, and undergo chairto-boat conformation changes. Paralleled with OXE cationic ring opening catalyzed by DBTDL and PUA to PUR conversions, these processes lead to self-repair by reformation of urethane and ether linkages. Although mechanistically and kinetically different, the self-healing outcomes with oxolane-chitosan (OXO-CHI) macromonomer in PUR networks are similar. Another interesting aspect is flow of displaced polymer mass.

Mechanical forces applied during damage alter the local free volume. As a result, the increase of localized free volume will favor chain mobility, thus aiding self-repairs. 
Thus, measurements of the local property changes on nano or micro level are desirable. As shown in Figure 6, A', following changes of physical properties within the damages during self-repair of OXE-CHI-PUR networks using AFM tips, two $\mathrm{T}_{\mathrm{g}} \mathrm{s}$ are identified at 61 and $102{ }^{\circ} \mathrm{C}$.[48] The lower $T_{g}$ corresponds to PEG-polyurethane, while the higher $T_{g}$ is due to OXE-CHI-urea phase. AFM measurements during repair show a repair front flow from the bottom up, followed by scratch closure upon further UV exposure.

Another approach of obtaining self-repairable PUR networks is by incorporating monosaccharide moieties into thermosetting PURs.[49] When methyl $\alpha$-Dglucopyranoside (MGP) is reacted with HDI and PEG to generate MGP modified polyurethane (MGP-PUR) network, self-repair occur at room temperature in air. Without $\mathrm{CO}_{2}$ and $\mathrm{H}_{2} \mathrm{O}$, however, it loses self-repairing property. Analysis of the damage-repair cycle of MGP-PUR revealed cleavages of ether and urethane linkages upon mechanical damage generating nucleophiles including $-\mathrm{OH}$ and $-\mathrm{NH}_{2}$ groups. This is depicted in Figure 6, B. During repair, MGP-urethane groups first complex with DBTDL assisted by the presence of $\mathrm{H}_{2} \mathrm{O}$, lowering $\mathrm{CO}_{2}$ insertion activation energy, after which, forming carbonate intermediates. The reactions of $-\mathrm{OH} /-\mathrm{NH}_{2}$ with carbonate intermediates result in the formation of carbonate and urethane linkages, thus repair the networks. This is the first study to utilize $\mathrm{CO}_{2}$ and $\mathrm{H}_{2} \mathrm{O}$ from the air to self-repair synthetic material, similar to carbon fixation of plants during photosynthesis, making synthetic material living like a plant. It should be noted that localized events measured by nanoindentation revealed initial modulus drop inside the damage, followed by the increase of the modulus until full recovery was reached. This is shown in Figure 6, B'. As a matter of fact, networks with variable morphologies and different $\mathrm{T}_{\mathrm{g}}$ f follow different patterns of physical recoveries. For example, thin films made from star polymers containing disulfide crosslinks on a silicon wafer exhibit surface tension driven viscoelastic flow behavior, [33] and selfhealing of poly(n-butyl acrylate) network containing alkoxyamine junctions shows enhanced mobility and flow[50]. These in-situ thermo/mechanical measurements at micro-scale reveal morphology changes of the scratches during repair, thus reveal the processes responsible for damage-repair cycle.

\section{Figure 6.}




\subsection{Supramolecular Chemistry}

Integrity of supramolecular material is achieved by the formation of non-covalent interactions.[51] This is typically accomplished by the presence of associative groups covalently attached to side chain or chain ends of the polymers, which bind the liquidlike polymers into a network with plastic or rubbery behavior resulting from non-covalent crosslinking. These interactions include hydrogen bonds, metal coordination, ionic interactions, $\pi-\pi$ stacking, and host-guest interactions. Several features make supramolecular chemistry particularly attractive when comes to self-healing: reversibility and speed, directionality, and sensitivity. In contrast to covalent bonding, these networks can be remodeled rapidly and reversibly from fluid-like to solid-like states. Due to the distinct nature of secondary bonds which differ from their polymer surroundings, the presence of associative groups usually induce morphology changes such as aggregation and crystallization. Thus, heterogeneities are inherent for many supramolecular networks affecting their physical/chemical properties, and facilitate self-repair. When mechanical force is applied, weaker supramolecular bonds will dissociate, and subsequent regeneration due to their dynamic character will allow self-repair.

\subsubsection{Hydrogen Bonds}

Although hydrogen bonds (H-bonds) are weaker than common covalent bonds, due to their directionality and affinity, a wide range of supramolecular polymers with diverse mechanical properties ranging from supramolecular gel to strong rubber like materials can be prepared. Concentration levels and the strength of H-bonds, length and rigidity of polymer backbone are the main factors determining mechanical strength and network remodeling. Several successful examples of self-repairing materials using $\mathrm{H}$ bonds have been developed and are shown in Figure 7.

\section{Figure 7.}

When fatty diacids and triacids were utilized in a two-step synthetic route involving condensation of acid groups with an excess of diethylenetriamine, followed by 
reactions with urea, a mixture of oligomers containing H-bonding motifs including amidoethyl imidazolidone, di(amidoethyl) urea, and diamido tetraethyltriurea is obtained. Associations of the H-bonding motifs leads to the formation of the self-repairing network shown in Figure 7, A.[52, 55] The resulting supramolecular assembly is a translucent glassy plastic which behaves like a soft rubber at temperatures up to $90{ }^{\circ} \mathrm{C}$, and is able to self-repair at ambient conditions. Although only single $\mathrm{T}_{\mathrm{g}}$ just below ambient temperature was observed, NMR measurements revealed substantial heterogeneities consisting of a mobile aliphatic phase and a rigid H-bonded phase.[56] While formation of H-bonds between amide $-\mathrm{C}=\mathrm{O}$ and amine-functionalized chains is responsible for network reformation, the presence of $\sim 15 \%$ mobile phase enables high healing efficiency at room temperature without any other stimuli. These heterogeneities are usually neglected due to the difficulty of detection, but in fact significantly affect network mobility. Notably, another interesting aspect of H-bonded networks is that self-healing reactions often compete with external conditions, such as the presence of moisture. For example, extended moisture exposure or heating of freshly cut specimens can significantly diminish the kinetics of self-healing due to the new equilibria,[57] which has been proven for the supramolecular network shown in Figure 7, A. Not only for supramolecular networks, but for most of the dynamic polymer systems, chemical activity and conformational changes immediately after mechanical damage play a key role in selfhealing.

The strength of directional multi-hydrogen bonds, such as triple H-bonds between thymine (Thy) and 2,6-diaminotriaine (DAT), quadruple H-bonds of ureidopyrimidinone (Upy), as well as multi-urea, are comparable to covalent bonds, giving the material soft rubber like properties. Taking advantage of high segmental mobility of polyisobutylenes (PIB), supramolecular PIB networks were developed containing various directional associative end groups offering variable dynamic behaviors, including self-repair.[58, 59] For example, PIBs functionalized with Thy and DAT end groups assemble into strong rubber like materials by formation of triple H-bonds as shown in Figure 7, B. Dynamic rheological studies revealed that enhanced mechanical strength is not simply due to the presence of triple-H-bonds, but is also attributed to network heterogeneity. Undirected supramolecular interactions between two or more Thy or DAT lead to complex cluster 
formation as shown in Figure 7, B, resulting in stronger crosslinked networks.[60] Taking advantage of such properties, a similar supramolecular PIB network containing barbituric acid moieties were tested for self-repairing.[53] These materials showed a rubbery plateau at high shear rates, and is self-repairable under ambient conditions by bringing the two physically separated parts into contact. Cluster formation is enhanced by polarity difference between associative chain ends and aliphatic main chains.

Due to the high dimerization constant of ureidopyrimidinone (Upy) units via quadruple H-bonds (Figure 7, C) supramolecular assemblies containing Upy show rubber-like behavior, and temperature dependent mechanical strength..[61-63] When Upy was incorporated into flexible poly(ethylene-co-butylene) and PDMS, aggregate formation of multiple Upy-dimers is responsible for increased mechanical strength and $T_{g}$ changes.[64, 65] For example, Upy-modified poly(ethylene-co-butylene) has two transitions at -57 and $69^{\circ} \mathrm{C}$, indicating the phase separation of aggregated Upy-dimers within amorphous poly(ethylene-co-butylene) matrix. Thermal activated dissociation and re-association at lower temperature enables repeatable self-repair, whereas soft polymer chains provide sufficient mobility after dissociation from the aggregate.[54, 66]

Due to strong association of Upy units poor solubility in most organic solvents is observed, making it difficult to process, thus requiring elevated temperatures. However if utilizing a multiphase polymers with soft and rigid domains with incorporated weaker Hbonds, can the H-bonds within the mobile phase increase self-healing efficiency? Such material can be synthesized from a brush copolymer comprised of rigid polystyrene (PS) backbone and soft polyacrylate amide (PA-A) brush.[13] The brush copolymers collapse into a core-shell nano-structure, where PS chains form the rigid core and PA-A side chains form the soft shell. H-bonds directed assembly through amide groups within the brushes lead to the formation of micro-phase separated microstructure, as shown in the TEM image in Figure 7, D. Young's moduli of resulting elastomer increased by a factor of three compared to the fatty acid based supramolecular network, while self-repair property at room temperature is maintained. Employing the same concept, different building blocks were utilized, such as poly(n-butyl acrylate) and polystyrene diblock copolymer (PBA-b-PS) end capped with Upy motifs at the PBA end yielding cylindrical microphase morphology[67]. Also, ABA triblock copolymers containing glassy 
poly(methyl methacrylate) (PMMA) and two dynamic poly(acrylate amide) (PA-amide) blocks will lead to core-shell spherical nanoparticles[68] which show self-healing attributes.

\subsubsection{Metal-Ligand Coordination}

Polymers containing metal centers emerged as easily processable materials with distinct optical properties and stimuli-responsiveness. The non-covalent binding between metal and macromonomers vary from strong irreversible to highly dynamic.[69-71] The latter has essentially been utilized in self-repairing metallosupramolecular networks, offering reversibility similar to H-bonds. Their photo responsiveness offers controllable self-repairing, while reversibility and tunability by incorporating different metal ion and ligand substitutes make coordination chemistry particularly attractive. It is distinct from $\mathrm{H}$-bonding that coordination number and conformations of the coordination center are tunable upon stimuli, and various structures can be obtained such as linear, star-shaped, highly branched, or dendritic. These characteristics are preferable for applications in dynamic self-repairing. Typical metal assisted self-repairing networks are illustrated in Figure 8.

It is well documented that organometallic conductive polymers comprising of $\mathrm{N}$ heterocyclic carbenes (NHCs) may exhibit corrosion control attributed to conductivity in

the order of $10^{-3} \mathrm{Scm}^{-1}$. Taking advantage of this property and the ability for coordinating with transition metals, formation of complexes utilizing NHCs and transition metals capable of self-repairs was developed.[72] Reversibility of the metal-polymer bonds is shown in Figure 8, A. When damaged, heating at $200{ }^{\circ} \mathrm{C}$ without solvent, or at $150{ }^{\circ} \mathrm{C}$ in the presence of DMSO vapor, will repair the damage due to the dynamic equilibrium between metal and the polymer, leading to flow into microcracks and reforming coordination bonds. The high repairing temperature is due to the strength of coordination bond and lack of soft segments.

Figure 8. 
Similarly, when competitive ligand hydroxyethylethylenediamino triacetic acid sodium salt (HEEDTA) was incorporated into (ruthenium) $\mathrm{Ru}$ (II)-bis-terpyridine crosslinked block copolymers, temperature changes decouple Ru metal from the ligand, resulting in bonding and de-bonding.[78] However, the binding strength of Ru (II)-bisterpyridine is comparable with a typical covalent bond,[79] which requires high energy to dissociate. Metal-ligand coordination can be tuned by choosing different metal ion and ligand pairs.[80] Similar networks, but coordinated through (Zinc) Zn (II)-bisterpyridine[73] and (iron) Fe (II)-bis-terpyridine[74] will result in self-repair, and the presence of heterogeneities will play a critical role during network rearrangements..

The optically healable supramolecular polymer based on amorphous poly(ethylene-co-butylene) core with 2,6-bis(1'-methylbenzimidazolyl)pyridine (Mebip) ligands at the termini can be crosslinked via $\mathrm{Zn}$ (II)-bis-terpyridine coordination. This is shown in Figure 8, B. [73] The presence of a hydrophobic segments and the polar metalligand motif results in phase separated morphology. Small-angle X-ray scattering (SAXS) and transmission electron microscopy (TEM) studies revealed the lamellar morphologies, where the metal-ligand complexes form a hard core that physically crosslinks the poly(ethylene-co-butylene) soft domains. The lamella spacing was $\sim 9 \mathrm{~nm}$. However, upon exposure to ultraviolet light, the metal-ligand motifs are electronically excited and the absorbed energy is converted into heat, causing temporary disengagement of the metal-ligand motifs, and a concomitant reversible decrease in the polymers' molecular mass and viscosity. The dissociation of the hard phase gives rise to the mobile macromonomers that will reform the original morphology, and metal-ligand coordination, facilitating healing of mechanical damages.

Similar metallosupramolecular system, although chemically different compared to $\mathrm{Zn}$ (II)-bis-terpyridine coordination, was developed using terpyridine- $\mathrm{Fe}^{2+}$ complexation pair with poly(alkyl methacrylate) as the main chain. This is shown in Figure 8, C.[74] Notably, in this particular system, phase separation results from the aggregation of ionic clusters. Also, instead of UV exposure, elevated temperature $\left(100^{\circ} \mathrm{C}\right)$ can be applied to repair damages. The metal-ligand bonds remain stable at this temperature, indicating that self-repair is not caused by dissociation of the coordination bonds, but attributed to 
reversible formation and dissociation of $6.4 \mathrm{~nm}$ ionic clusters triggered by the presence of $\mathrm{Fe}^{2+}$.

There is increasing evidence that metal-ligand coordination play an important role in the dynamics of biological rearrangements. Recent studies took advantage of coordination between Fe (III) and catechol ligands that mimic mussel adhesives in nature, which resulted in $\mathrm{pH}$-induced crosslinked self-healing polymer with near-covalent elastic moduli.[75, 81-83] The networks are formulated from 3,4 dihydroxyphenylalanine (DOPA) modified poly(ethylene glycol) (PEG) or polyallylamine. The uniqueness of this approach is the ability to switch from mono-, bis, to tris-Fe-catechol complexes at different $\mathrm{pHs},[75]$ thus providing the ability of crosslinking control without $\mathrm{Fe}$ (III) precipitation. Figure 8, D depicted reversible tris-Fe(III)-catechol complex formation. Similarly, crosslinked hydrogels were also prepared by complexation of branched cathehol derivatized poly(ethyleneglycol) (PEG) with 1,3-benzenediboric acid. [84, 85] Later on, a heterogeneous structure inspired by mussel was developed. Peptide amphiphile molecules composed of a hydrophobic lauryl group attached to the Nterminus and DOPA attached to the opposite end of the peptide segment were synthesized. [76] Analogous to mussel byssus, the amphiphilic molecules aggregate into nanofiber structures with hydrophilic peptide sequence containing DOPA exposed to aqueous environment. Interfiber crosslinking by complexation of Fe (III) and DOPA at $\mathrm{PH} \approx 10$ enhanced the mechanical strength of the network, while dissociation of tris$\mathrm{Fe}$ (III)-catechol bonds create free moving fibers. Self-repair occur upon rearrangement of the nanofibers and reformation of crosslinks. Figure 8, D shows TEM image of the nanofibers crosslinked by tris-Fe-catechol. This strategy demonstrated the adaptability of metal binding to various complex supramolecular networks enabling self-repair while enhancing the mechanical strength without disturbing the original morphology.

Facile UV-healable polyethylenimine-copper $\left(\mathrm{C}_{2} \mathrm{H}_{5} \mathrm{~N}-\mathrm{Cu}\right)$ supramolecular polymer networks were also developed. The uniqueness of this network is that selfhealing is not only induced by reversible formation of $\mathrm{C}_{2} \mathrm{H}_{5} \mathrm{~N}-\mathrm{Cu}$ complexes, but also square-planar-to-tetrahedral conformational changes of the coordination center induced by the charge transfer between $\sigma(\mathrm{N})$ bonding and $\mathrm{dx}_{x^{2}-y^{2}}(\mathrm{Cu})$ antibonding orbitals upon UV absorption.[77] The structural and conformational changes of the complex center are 
shown in Figure 8, E. The potential use of a hybrid polymeric scaffold, in which covalent crosslinked permanent scaffold co-existed with reversible metal-ligand coordinative crosslinks were also investigated.[86] This study utilized a covalent gel polymerized from hydroxyl-2-3thylmethacrylate (HEMA), 4-vinylpyridine (4VP), and a bis-functional corsslinkeder ethyleneglycol dimethacrylate (EGDMA), which is a permanently crosslinked network. The transient crosslinks were generated by adding bifunctional $\operatorname{Pd}($ II) or Pt( II) pincer complexes, which form crosslinks with the polymer side-chain pyridine ligand through metal-ligand coordination. Dissociation and reassociation of the transient crosslinks were manifested by an increase of the storage modulus G' with increasing frequency. The advantage of the hierarchical design is toughness while enabling "programmable" macroscopic mobility. A self-repairing polydimethylsiloxane/polyethylene glyco-polyurethane (PDMS/PEG-PUR) Cu-catalyzed networks in which $\mathrm{Cu}-\mathrm{O}$ coordination was embedded into covalently crosslinked polyurethane networks were developed.[87] In addition to $\mathrm{UV}$ induced $\mathrm{Cu}-\mathrm{O}$ bond reformation along with conformational changes, cleavage and reformation of covalent $\mathrm{Si}$ O linkages were detected, indicating a multi-level repair process. Nanoindentation measurements indicated the increase of modulus inside the scratch immediately after damage. This was attributed to the formation of $\mathrm{CuCl}_{2}$ clusters resulting from $\mathrm{Cu}-\mathrm{O}$ bond cleavage. The interfacial property and heterogeneity at the newly created surfaces upon damage is another area that is critical to self-repair, but has not been extensively studied.

\subsection{3 $\pi-\pi$ Stacking Interactions}

$\pi-\pi$ stacking interactions achieved by end-capped $\pi$-electron-deficient groups interacting with $\pi$-electron-rich aromatic backbone molecules were utilized in the development of thermal-triggered reversible self-healing supramolecular polymer networks. This was achieved by employing chain-folding copolyimide (electron deficient) and pyrenyl (electron rich) end-capped polysiloxane[88] or polyamide[89] chains. The $T_{g}$ of the network can be tuned to achieve self-healing at relatively wide temperature range $(\sim 50$ $100^{\circ} \mathrm{C}$ ) by changing the spacer and adjusting the composition of the blend. Upon heating, the $\pi$ - $\pi$ stacking interactions will be interrupted, enabling pyrenyl end-capped chains to disengage from copolyimide and flow due to the presence of a flexible "soft" spacer. 
Thus, repair of damage and regaining of mechanical strength by reformation of the $\pi-\pi$ stacking will occur. Other approaches combined $\pi-\pi$ stacking with intermolecular Hbonding within one supramolecular network to gain efficient thermal healability using polybutadiene containing urethane and urea groups as spacer.[90] Networks with enhanced mechanical properties were produced using tweezer-type bis-pyrenyl end groups instead of mono-pyrenyl groups.[91] Figure 9 illustrates the formation of tweezer type interactions between bis-pyrenyl end groups (compound $\mathbf{1}$ ) and naphthalene-diimide (compound 3) chains. Non-tweezer type interactions using mono-pyrenyl groups (compound 2) are also shown. The [1+3] blend exhibited higher tensile modulus and elongation compared to [1+2] blend, as summerized in Figure 9, although requires higher healing temperature and longer time. Nitrobenzoxadiazole (NBD)-containing cholesterol (Chol) derivatives also offer a unique combination of $\pi-\pi$ stacking and intermolecular $\mathrm{H}$ bonding interactions,[92] where the length of the spacer connecting NBD and Chol units is critical in controlling gel and healing properties.

\section{Figure 9.}

\subsubsection{Ionic Interactions}

Ionic interactions in polymers are primarily manifested by the formation of ionomers,[93] and selected polymers demonstrated self-healing attributes. For example, in poly(ethylene-co-methacrylic acid) (EMAA) shown in Figure 10, A, polymer diffusion in a damaged area occurred under ambient and elevated temperatures upon projectile puncture testing.[94] A ballistic puncture in low density polyethylene (LDPE) did not show healing, whereas puncture in EMMA films healed the puncture leaving a scar on the surface. The proposed healing mechanism is a two stage process in which upon projectile impact, ionomeric network is disrupted, and heat generated by the friction during the damage is transferred to the surroundings, generating localized melt state. The molten polymer surfaces fuse together via inter-diffusion to seal the puncture, followed by reshuffling of the ionic clustered regions and relaxation.[95] Because self-healing in this case largely relies on thermo activated chain diffusions, increased cluster sizes that immobilize the chains will reduce the healing rate.[96] However, if the clusters are able 
to dissociate upon stimuli, this process may enhance the healing rate. This was demonstrated by using polyelectrolytes synthesized from poly(acrylic acid) (PAA)/poly(allylamine hydrochloride) (PAH) pairs shown Figure 10, B. By applying ultracentrifugation and extrusion in the presence of salt yielding compact polyeletrolyte complexes (CoPECs), this systems exhibited self-healing attributes.[97] The self-healing ability becomes stronger with increasing concentrations of $\mathrm{NaCl}$ salt, indicating that dynamics of the material originates from interrupting ionic interactions between the polyelectrolyte pairs by salt, which increase the chain mobility. The effect of ionic concentrations on self-healing of EMMA ionomers[98] revealed the relationship between elasticity, diffusion, and the self-healing behavior at various temperatures. Increased ionic content produces higher crosslinking density, but less mobile polymer structures, which yield an increased resilience and elasticity in the melt not observed in non-ionic varieties. It created a much stronger network surrounding the impact site to allow the needed elastic response to repair. The non-ionic variations loose healing ability upon reaching $\mathrm{T}_{\mathrm{m}}$. High concentrations of ionic species are detrimental to low temperature healing due to the rigidity. Such relations between elasticity, diffusivity, healing temperature, and self-healing ability are not limited to ionomers, but also applicable to other self-healing systems.

Initiated by the presence of water, polyelectrolyte multiplayered assemblies have demonstrated the ability to recover from mechanical deformations.[99, 100] Under dry conditions, the Young's modulus is as high as $11.8 \mathrm{GPa}$, but can be reduced to $0.44 \mathrm{MPa}$ in the presence of $\mathrm{H}_{2} \mathrm{O}$ resulting from hydrophilic nature of the ionic pairs. The role of multilayered structures in the presence of ionic interactions was not addressed.[101] Stiff and self-healing gels were also developed from complexation of polyamines with phosphate-bearing multivalent anions, which are shown in Figure 10, C.[102] They exhibited strong underwater adhesion to both hydrophilic and hydrophobic surfaces as well as self-healing through reversible ionic crosslinks. Another water induced selfhealing system, although based on H-bonds instead of ionic interactions, was synthesized from polyacrylate and polymethacrylate polymers surface-functionalized with musselinspired catechols.[103] The catechol groups are protected by triethylsilane. Immersing the cut samples in water at $\mathrm{pH}=3$ removes the triehtylsilyl protecting groups at the cut, 
thus activating chatochol groups and subsequent rejoining of the cut surfaces by the formation of intermolecular $\mathrm{H}$-bonds between catechol and oxidized quinones.

\section{Figure 10.}

\subsubsection{Host-Guest Interactions}

Host-guest interactions refer to the targeted binding of two structurally dissimilar but dimensionally matched macromolecular units via weak interactions including $\mathrm{H}$ bonding, $\pi-\pi$ interactions, van de waals, or hydrophobic interactions. While naturally existing host-guest assemblies exhibit great biological importance, synthetic host-guest pairs capable of reversible guest exchanges[104, 105] may facilitate the formation of selfrepairable supramolecular gels. Taking advantage of cyclodextrin's (CD) cavity capable of entrapping other molecules, host-guest interactions between CD (host) and polyacrylic acid terminated with ferrocene (guest) were utilized to create hydrogels.[106] Redox stimuli induced a sol-gel transition, and dissociation/re-association between CD and ferrocene facilitate the self-repair. Not surprisingly, supramolecular hydrogels prepared from polyacrylamide modified with $\mathrm{CD}$ crosslinked with aliphatic guest groups via hydrophobic interaction also showed repairing upon rejoining the ruptured surface. [107] Similar supramolecular hydrogels were also prepared from crown ether and bisammonium based poly(methyl methacrylate) (PMMA) polymers. Macrocyclic host molecule cucurbit[8] uril ( $\mathrm{CB}[8])$ capable of reversible cross-linking with multivalent copolymers with high binding constants $\left(\mathrm{Ka}>10^{11}-10^{12} \mathrm{M}^{-2}\right)$ was also shown to have the potential to be utilized in self-healing materials.[108] In summary, numerous unexplored opportunities in supramolecular hydrogels based on host-guest interactions exist for the development of 3D structures.

Another group of reversible and potentially self-healable materials that have promising properties in bioengineering applications are based on DNA or protein crosslinks. One example are the proteins that consist of terminal leucine zipper which demonstrated reversible gel formation controlled by mild $\mathrm{pH}$ and/or temperature variations.[109, 110] Taking advantage of the dynamics of 'living' biomacromulecules and combining them with synthetic macromolecules, DNA strands were utilized to 
connect polymer chains to form crosslinkable networks which can be disrupted by adding unassociated DNA “fuel” strands.[111-115]

In view of the above discussion, polymer networks that rely on reversible covalent bonds and secondary interactions may offer self-repairs in which bond dynamic and chain mobility affect self-repairing efficiency. A common denominator of many studies is that rationally designed heterogeneities not only facilitate distribution of hard or soft segments and free volume, but also provide bulk integrity. Reversible bonds incorporated into hard-soft domains or their interfaces may enhance dissociation or reposition of soft domains while maintaining local integrity during repair. If designed properly, heterogeneous polymeric materials with glassy or tough rubber-like properties may offered high repair efficiency under ambient conditions. Heterogeneous materials that may also offer self-repair properties are nanocomposites. Owning to large interfacial regions, design of reversible interfacial chemistry may offer unique opportunities for development of new materials. The next sections will examine the role of interfaces in multi-phase composites and their potential impact on self-healing.

\section{Nanocomposites and the Role of Interfaces}

While the presence of heterogeneities at macromolecular scales plays a significant role in facilitating segmental mobility and rearrangements during repair, the question is whether anisotropic rigid components are capable of generating self-healing in composites.. Intuitively, rigid nanoparticles, nanotubes, or fibers will not contribute to self-repair, unless they have inherently built-in self-healing components. However, if interfacial regions between polymer matrix and reinforcing rigid entities exhibit the ability of bond cleavage and reformation, interfacial regions may serve that purpose. The majority of nanoparticles, nanorods/nanowires, nanotubes, nanofibers, and graphene sheets can be surface modified to generate amine,[116] carboxylic acid,[117] hydroxyl,[118] UPy,[119] thiol,[120] furfuryl,[121] cinnamoyl,[122] anthracene,[123] or pyrenyl groups[124], which can react with their counterparts in the matrix. Thus, the nature of modifications will be determined by the polymer motif to form reversible bonds with a polymer matrix. Consequently, interfacial chemistry will be critical in achieving reversible self-repair. Figure 11 summerizes commonly utilized nanomaterials (A) and 
their surface functionalizations (B). For surface functionalization to be effective, polymer matrix should consist of "matching" reactive groups, which are illustrated in column C. Thus, when $\mathrm{B}$ and $\mathrm{C}$ react, interfacial bonding in column $\mathrm{D}$ will facilitates covalent and non-covalent interfacial interactions. One can envision that reforming bonding at the fiber-matrix interface may facilitate repairs when the interface undergoes partial damagerepair cycle, but overall mechanical integrity of a composite is retained. When a load is removed, the reformed interface will return to equilibrium conditions. This is schematically illustrated in Figure 11, E.

\section{Figure 11.}

An illustrative example of organic-inorganic hybrid compound is polyhedral oligomeric silsesquioxane (POSS, with empirical formula of $\mathrm{R}_{\mathrm{n}}\left(\mathrm{SiO}_{1.5}\right)_{\mathrm{n}}, \mathrm{n}=8,10$, or 12), where $\mathrm{R}$ groups on each cage silicon atom can be either reactive or nonreactive organic groups. The cubic cage can be considered as the smallest possible nanoscale particles of silica.[125-127] Figure 12 shows thermally healable POSS composite based on DA reactions.[128] Compared with $\mathrm{SiO}_{2}$ nanoparticles functionalized with furfuryl functional groups,[121] POSS composites exhibit a modulus of $1.0 \mathrm{GPa}$ and a yield strength of 118 $\mathrm{MPa}$. Owing to the molecular level dispersion of POSS nanocage in the composite system, self-healing of a crack can be effectively achieved at $135-150{ }^{\circ} \mathrm{C}$ within 30 min. Since POSS with glycidyl reactive groups or other reactive ends can be easily converted into desired functional groups, one can envision functionalization of POSS with other stimuli-responsive reactive moieties, such as amine, carboxylic acid, hydroxyl, UPy, thiol, furfuryl, cinnamoyl,[102] anthracene, or pyrenyl groups shown in Figure 11.

\section{Figure 12.}

Stimuli-responsive reactive moieties can be also introduced onto nanoparticle surfaces by "grafting to" (i.e. $-\mathrm{OH},-\mathrm{NH}_{2},-\mathrm{COOH},-\mathrm{SH}$ ) or "grafting from" technique (i.e. initiator-functionalized nanoparticles).[129] Nanoparticles modified with alkane chains may improve to a certain extent interfacial interactions with polymer matrices, 
enhancing phase compatibility and mechanical properties. However, as interfacial interactions between nanoparticles and polymer matrices are typically manifested by hydrogen bonding, electrostatic, or van der Waals interactions, there is a possibility of aggregate formation when nanocomposites are subjected to cycling loading or temperatures.[130] Ultimately, modifying nanoparticles with responsive functional groups such as cinnamoyl, pyrenyl and furfuryl groups, forming reversible covalent linkages with polymers matrices, may provide an opportunity for photo or thermotriggered self-healing.[121, 122, 124]

Figure 13 illustrates an example of pyrene functionalized gold nanoparticles (AuNPs) and pyrene end-capped polyamide (as $\pi$-electron donors) interacting with polydiimide (as $\pi$-electron acceptor).[131] Self-healing is achieved due to formation of thermally reversible $\pi-\pi$ stacking interactions between functionalized nanoparticles and the polymer matrix. Compared with regular ligand functionalized AuNPs, pyrenefunctionalized AuNPs are uniformly distributed and a linear increase of tensile moduli and tensile strength were observed.

\section{Figure 13.}

Of particular interest is the design of remote self-healing nanocomposites. The concept involves the utilization of remote sources of energy, such as electric and magnetic fields, and converting remote stimuli into localized heat in damaged area. As a result, mobility of polymer chains or segments is enhanced, thus inducing interfacial diffusion to facilitate self-repairs. This formulated the idea of using superparamagnetic $\gamma$ $\mathrm{Fe}_{2} \mathrm{O}_{3}$ nanoparticles that oscillate in the presence of oscillating magnetic field. This is schematically shown in Figure 14, where $\gamma-\mathrm{Fe}_{2} \mathrm{O}_{3}$ nanoparticles were incorporated into a thermoplastic polymer network.[132] The oscillating motions of the nanoparticles convert magnetic frequency into heat at a nanoparticle/polymer interface, which facilitates polymer diffusion to permanently repair the crack. Such process can be repeated multiple times. Similarly, noble metal nanoparticles such as AuNP and AgNP have also been utilized to remotely trigger repairs by generation of surface plasmon 
resonance, which converts incident light (usually near infrared spectrum) into local heat.[133-139]

\section{Figure 14.}

Nanorods (organic or inorganic nanorods) are popular nanomaterials exploited in self-healing composites to enhance mechanical properties as well as introduce healing attributes. Dispersions of cellulose nanorods in polymer matrix have been reported to enhance the mechanical strength, while self-healing process may be governed by $\pi-\pi$ stacking interactions only occurring within the polymer matrix.[140] Due to lack of effective interfacial bonding between nanorods with polymer matrix, phase separation occurred when the nanorod concentration was more than $10 \mathrm{wt} \%$, leading to compromised mechanical strength and healing efficiency. Similar to POSS, cellulose which contains abundance of hydroxyl groups can be modified to enhance both mechanical properties and self-healing. As shown in Figure 15, cellulose nanocrystals (CNC) carrying numerous hydroxyl groups $(-\mathrm{OH})$ are reacted with ureidopyrimidone (UPy-NCO) to form strong UPy-UPy hydrogen-bonding interactions with the polymer matrices which also contain UPy motifs.[119] The UPy motif exhibits UV absorption at wavelength of $320-390 \mathrm{~nm}$ with intensity of $250-350 \mathrm{~mW} / \mathrm{cm}^{2}$, thus converting to heat, will trigger self-healing process. Exposure as short as 20-80 s is sufficient for repairs to occur. Inspired by this concept, nanorods possessing optoelectronic properties (transition metal oxide nanorods, noble metal nanorods) $[141,142]$ may also be functionalized with photo-responsive functional groups (i.e. UPy, cinnamoyl, anthracene) to create nanocomposites with optoelectronic responses and remotely controlled healing performance.

\section{Figure 15.}

The concept of heterogeneities based on host-guest interactions discussed above was also utilized to fabricate self-healable nanocomposites. Although one can envision deficiencies of the host-guest approach, their primary advantage is rapid response. For 
example, $\mathrm{CB}[8]$ macrocyclic host molecules discussed in Section 2.2 were paired with methyl viologen(MV)/naphthyl(Np) to design a cellulose nanorod reinforced composite hydrogels with three-component recognition.[108, 143] As shown Figure 16, supramolecular composite hydrogel comprised of (A) soft poly(vinyl alcohol-co-methyl viologen) (PVA-co-MV) containing the first guest MV, (B) hard reinforcing cellulose nanocrystals (CNC) grafted with poly(dimethylaminoethyl methacrylate-co-naphthyl methacrylate) (CNC-g-PDMAEMA-co-NpMA) containing the second guest $\mathrm{Np}$, and (C) host $\mathrm{CB}[8]$ was developed.[144] Combining these components in aqueous environments, a supramolecular network is formed by binding with the MV and Np guest motifs with host $\mathrm{CB}[8]$. Rheological measurements revealed the highest storage modulus up to 14.3 $\mathrm{kPa}$ and the fast sol-to-gel transitions as well as rapid self-healing (ca. 6s). These features of a nanocomposite hydrogel are particularly useful for applications in biomedical fields to overcome brittleness of conventional hydrogels and to impart with rapid recovery of mechanical properties.

\section{Figure 16.}

Carbon nanotubes (CNT) and graphene nanosheets (GNS) are known for their unique physical properties, such as high mechanical strength, large surface area, and $\pi-\pi$ conjugated structures that offer remarkable electric properties.[145] They are combined with various polymers to offer reinforced strength and integrated properties.[146] Taking advantage of their unique electromagnetic properties, CNT and GNS have been utilized in developing nanocomposites for remotely triggered self-healing. Amide functionalized single-walled CNTs (SWNTs) uniformly dispersed in protonated Nafion SMP exhibited repair properties by IR radiation.[147] Graphene reinforced polyurethane (PUR) composite also exhibited healing attributes.[148] Self-healing was achieved by graphene's ability to convert IR irradiation, electromagnetic wavelength, or electricity into heat, enhancing PUR chain mobility and re-entanglement at the damaged interfaces, resulting in remotely-triggered multi-responsiveness. Both CNT and GNS can be chemically modified by partial oxidization to introduce carboxylic acid groups, followed by cross-linking with polymer matrix, or further modifications with other functionalities 
to enhance dispersiveness and self-healing responses.[117] The main limitation is the tendency to over oxidize that leads to lower optical absorption and electric conductivity. In order to retain opto-electric properties, phenylhydrazine was used to reduce graphene oxide to recover near infrared (NIR) absorption after graphene oxide modification with phenyl isocyanate. [149] Although CNT and GNS can be directly used to fabricate potentially healable nanocomposites, special fabrication techniques may be advantageous. For example, aligned-CNT sheets were prepared by dry-drawing from a spinnable CNT array, which were then wrapped around self-healable polymer fibers to give an electrically conductive wire with uniformly attached CNT sheet shell onto the polymer fiber core.[150] The aligned-CNT sheet showed enhanced conductivity, lower electrical resistance, and recovery of capacitance after self-healing.

Besides nanoparticles, nanorodes, carbon nanotubes, and graphene nanosheets, carbon and glass fibers as well as metal wires are perhaps the most commonly utilized components in fabricating fiber reinforced healable composites. When developing fiber reinforced self-healing composites proper fabrication techniques are critical to uniformly disperse anisotropic components. For example, a silver nanowire reinforced healable conductor was constructed by drop-casting a layer of silver nanowire solution on a substrate surface, followed by deposition of co-monomers solutions bearing DA reactive groups on top of the silver nanowire layer, which was then cured at $70{ }^{\circ} \mathrm{C}$ to give the healable conductive composite based on DA chemistry.[151] Several strategies for interfacial healing of fiber/polymers have been reported. For example, glass fiber was functionalized with maleimide-groups to recover interfacial bonding with a furanfunctionalized epoxy matrix by heat treatment.[152] Along the same line, carbon or glass fiber surfaces were bound with micro or nanocapsules loaded with healing agents and were incorporated into polymer matrix. Once the interfacial damage occurred, the capsule is cracked and healing agent is released to re-bond the fiber and polymer matrix, though this method is limited by un-repeatable healing.[153] Microcapsule-based delivery of healing agents is limited to one time use, but certain advantages for interfacial healing of fiber/polymer matrix are feasible.

\section{Physical Aspects of Self-Healing}


Physical aspects of self-healing are driven not only by chemical and morphological features of polymer or composite networks, but also by the modes of damage in a given environment. Earlier concepts of self-healing came out from two physical observations: (1) microcrack formation upon mechanical loading, followed by the recovery to the original elastic properties;[154] several recent studies demonstrated the dynamics of reversible bonds during self-healing by monitoring the decay of mechanical properties after multi-cycle load and rest. (2) self-healing upon projectile puncture, as observed in poly(ethylene-co-methacrylic acid)copolymers (EMMA).[95, $155,156]$ In view of these earlier observations, dynamics of mechanical damage and dissipation of thermal energy during damage were considered the primary sources of repairs. For example, the energy impact during the projectile melted the polymer at the damage zone, allowing inter-diffusion to self-heal. Several models were established at that time to explain the underlying physics governing this behavior summarized in recent literature.[2]

Initial studies on thermoplastic polymers suggested that self-healing of a crack involves five stages: segmental surface rearrangements, surface approach, wetting, diffusion, and randomization.[157], In reality, different damages will occur in different materials resulting in complex damage morphologies. Neglecting material loss during damage, Surface rearrangements and surface approach to fill the voids are critical in order to diffusion or elastic recovery as demonstrated in several studies. [33, 46, 47] The question how surface topography variations will impact materials recoveries is not well understood. Furthermore, wetting, defined as a formation of the polymer-polymer or polymer-liquid interfaces prior to diffusion, was considered as prerequisite to regain mechanical properties at the repaired area via chain entanglements. In this context, the reptation model[158] semi-quantified these events by describing the motion of macromolecular segments trapped in a stationary tube and self-healing was achieved only when the entire chain completely disengaged from the tube, reaching equilibrium state after a given time $T_{r}\left(T_{r}\right.$ is the time required for complete chain disengagement from the tube). Thus, self-repairing process can be viewed as chain conformational changes going from non-Gaussian to equilibrated Gaussian state during which each chain escapes from the tube cavity in a given time. As expected, longer chains would take longer to escape, 
and this approach provided the molecular weight (M) dependence of repair time $T_{r} \propto M^{3}$, implying that lower molecular weight polymers exhibit favorable repairing conditions. Assuming no bond cleavage during damage, chain diffusion was the only driving force for repair, and the source of loose chain ends was chain slippage. In this context, healing efficiency $R(\sigma)$ can be defined as the ratio of fracture stress before $\left(\sigma_{\text {initial }}\right)$ and after healing $\left(\sigma_{\text {healed }}\right)$. Assuming the reptation mode of polymer diffusion, it has been shown that the fracture stress is $M$ and $t$ dependent via $\sigma_{\propto}(t / M)^{1 / 4}$ relationship (where: $t$ is the repairing time). If during time $t$, a chain escapes the tube at $T_{r}$, and repair is completed, then $t=T_{r}$. This would imply that only conformation changes result in self-healing. Conceptually, if repair is considered as the rearrangement of chain conformations from non-Gaussian to Gaussian states, the time required for chains to escape the tube as well as the recovery of fracture stresses will favor low molecular weight polymers, as they exhibit enhanced chain mobility and shorter repairing times as well as energetically favorable stress recoveries. While the $\sigma \propto \mathrm{t}^{1 / 4}$ dependence has been experimentally shown for induced crack healing in poly(methyl methacrylate) (PMMA)[159] as well as theoretically predicted[160,161]. This raises the question about the role of the polymer glass transition temperature $\left(\mathrm{T}_{\mathrm{g}}\right)$ on self-healing when bond cleavage occurs. Our recent studies show that self-healing is partially due to the formation of a mobile layer at the newly formed interface resulting from the presence of either shorter chains due to chain cleavage, or chain slippage resulting in dangling ends with one end anchored to the polymer matrix, or untangled short chains with two ends free.. The latter will be strongly affected by copolymer topographies and copolymer compositions. As described by a selfhealing lattice model,[5] the shorter chains create favorable entropic conditions for chain rearrangements. The chains will self-repair as the bonds reform and the chain length will increase to reach a new equilibrium state. In addition, strain release and higher free volume also contribute to lowering $\mathrm{T}_{\mathrm{g}} \mathrm{s}$ at the polymer-air interface, as demonstrated in previous studies.[162-164] The chains mobilities below the $T_{g}$ were also described by a so-called 'twinkling' fractal theory[165] which assumed that at any temperature, a fraction of the solid and liquid atoms forming interfacial clusters coexists in an equilibrium state described by the Boltzmann distribution of energetically different 
levels. The term 'twinkling' implied that a fractal structure of liquid and solid atoms exchange energy with a frequency spectrum $\mathrm{F}(\omega)$.

Experimentally demonstrated decrease of the $T_{g}$ in damaged areas have been shown on polyurethanes, $[47,48]$ which are able to recover the $T_{g}$ as a result of increased crosslink density during self-healing. Furthermore, the role of heterogeneities in the interfacial regions outlined in Sections 3 and 4 illustrated that the simultaneous presence of hard (high $\mathrm{T}_{\mathrm{g}}$ ) and soft segments (low $\mathrm{T}_{\mathrm{g}}$ ) may facilitate self-healing. Thus, the prerequisite that the low $T_{g}$ will facilitate self-healing is not necessarily valid. On the contrary, there is increasing evidence that the presence of heterogeneities at molecular and mesoscale scale lengths also facilitates self-repairs. The simultaneous presence of hard (high $T_{g}$ ) and soft segments (low $T_{g}$ ) may lead to self-healing of phase-separated polymers. The evolution of this concept has led to another class of self-healing materials with so-called shape memory components. These components exhibit spring-like behavior, which upon mechanical damage and/or compression go back to their initial state. As one would anticipate, shape memory components will facilitate faster repair due to elastic, spring-like responses, whereas interfacial flow and 'filling up' a wound may be kinetically slower. Thus, in designing self-healing polymers it is critical to adjust stability of reactive groups (for covalent and supramolecular rebonding) with kinetics of interfacial flow or responses of shape memory components. For diffusion-driven rebonding, maintaining fluid-like mobility near the interfacial regions will be critical. This is schematically depicted in Figure 17, A and B.

\section{Figure 17.}

\section{Conclusions and Insights}

Materials' abilities to mimic self-healing of living organisms are of great significance in prolonging lifespan and efficient utilizations of resources and energy. Driven by the technological needs, several successful approaches have been explored over the last decade. Incorporating stimuli-responsive components, such as dynamic covalent bonds and supramolecular chemistries into polymers with intentionally introduced heterogeneities offer highly effective strategies in the design of materials of 
the $21^{\text {st }}$ century. Heat, electromagnetic radiation, environment changes, or the presence of atmospheric $\mathrm{CO}_{2}$ and $\mathrm{H}_{2} \mathrm{O}$ offers external stimuli to generate favorable thermodynamics and kinetics conditions for repair. While the majority of the studies focused on the chemistries of self-healing, physical aspects are of the equal significance. Numerous examples discussed in this review point towards the significant contributions of heterogeneities and their distributions on self-repair of polymer materials. Combining hard and soft segments into one polymer network, or reinforcing with nano-objects containing desirable interfacial chemistries will pave the path to a new generation of materials with life-like attributes. Notably, one reaction usually will not be sufficient to facilitate network repairs, just like not only one single chemical bond will cleave during mechanical damage. It is the combination of orchestrated physical-chemical events that facilitate unique self-healing properties. These inherent properties make self-healing a challenging task. Although chemical imaging may provide useful molecular level information regarding chemical processes involved in network repairs, understanding of mechanical properties along with morphological information on small scales are also critical. Clearly, there is a need for combining chemical analysis with localized mechanical testing in order to advance our knowledge in this critical field. Future studies should take advantage of the concept of heterogeneities in achieving rapid stimuliresponsiveness of rigid materials as well as multi-level chemical interactions achieved by synchronized chain movements and repair of multiple type chemical entities within one material.

\section{Acknowledgements}

The authors thank the National Science Foundation under award CMMI 1332964 and J. E. Sirrine Foundation Endowment at Clemson University for partial support of these studies, 


\section{References}

[1] Wu DY, Meure S, Solomon D. Self-healing polymeric materials: a review of recent developments. Prog Polym Sci 2008;33:479-522.

[2] Wool RP. Self-healing materials: a review. Soft Matter 2008;4:400-18.

[3] Murphy EB, Wudl F. The world of smart healable materials. Prog Polym Sci 2010;35:223-51.

[4] Hager MD, Greil P, Leyens C, van der Zwaag S, Schubert US. Self-Healing Materials. Adv Mater 2010;22:5424-30.

[5] Yang Y, Urban MW. Self-healing polymeric materials. Chem Soc Rev 2013;42:7446-67.

[6] Vaiyapuri R, Greenland BW, Colquhoun HM, Elliott JM, Hayes W. Evolution of supramolecular healable composites: a minireview. Polym Int 2014;63:933-42.

[7] Eyring H. Plasticity, and Diffusion as Examples of Absolute Reaction Rates. J Chem Phys 1936;4:283-91.

[8] Flory PJ, Krigbaum W. Statistical mechanics of dilute polymer solutions. II. J Chem Phys 1950;18:1086-94.

[9] Simha R, Boyer RF. On a General Relation Involving the Glass Temperature and Coefficients of Expansion of Polymers. J Chem Phys 1962;37:1003-7.

[10] Liu F, Urban MW. Recent advances and challenges in designing stimuliresponsive polymers. Prog Polym Sci 2010;35:3-23.

[11] Liu F, Urban MW. New thermal transitions in stimuli-responsive copolymer films. Macromolecules 2009;42:2161-7.

[12] Liu F, Jarrett WL, Urban MW. Glass (Tg) and Stimuli-Responsive (T SR) Transitions in Random Copolymers. Macromolecules 2010;43:5330-7.

[13] Chen Y, Kushner AM, Williams GA, Guan Z. Multiphase design of autonomic self-healing thermoplastic elastomers. Nat Chem 2012;4:467-72.

[14] Wright WJ, Hufnagel T, Nix W. Free volume coalescence and void formation in shear bands in metallic glass. J Appl Phys 2003;93:1432-7. 
[15] White SR, Sottos NR, Geubelle PH, Moore JS, Kessler MR, Sriram SR, Brown EN, Viswanathan S. Autonomic healing of polymer composites. Nature 2001;409:7947.

[16] Chen X, Dam MA, Ono K, Mal A, Shen H, Nutt SR, Sheran K, Wudl F. A Thermally Re-mendable Cross-Linked Polymeric Material. Science 2002;295:1698-702.

[17] Chen X, Wudl F, Mal AK, Shen H, Nutt SR. New Thermally Remendable Highly Cross-Linked Polymeric Materials. Macromolecules 2003;36:1802-7.

[18] Zeng C, Seino H, Ren J, Hatanaka K, Yoshie N. Bio-Based Furan Polymers with Self-Healing Ability. Macromolecules 2013;46:1794-802.

[19] Bai N, Saito K, Simon GP. Synthesis of a diamine cross-linker containing DielsAlder adducts to produce self-healing thermosetting epoxy polymer from a widely used epoxy monomer. Polym Chem 2013;4:724-30.

[20] Liu YL, Chen YW. Thermally Reversible Cross-Linked Polyamides with High Toughness and Self-Repairing Ability from Maleimide- and Furan-Functionalized Aromatic Polyamides. Macromol Chem Phys 2007;208:224-32.

[21] Barthel MJ, Rudolph T, Teichler A, Paulus RM, Vitz J, Hoeppener S, Hager MD, Schacher FH, Schubert US. Self-Healing Materials via Reversible Crosslinking of Poly(ethylene oxide)-Block-Poly(furfuryl glycidyl ether) (PEO-b-PFGE) Block Copolymer Films. Adv Funct Mater 2013;23:4921-32.

[22] Oehlenschlaeger KK, Mueller JO, Brandt J, Hilf S, Lederer A, Wilhelm M, Graf R, C ML, Schmidt FG, Barner-Kowollik C. Adaptable Hetero Diels-Alder Networks for Fast Self-Healing under Mild Conditions. Adv Mater 2014;26:3561-6.

[23] Chung C-M, Roh Y-S, Cho S-Y, Kim J-G. Crack Healing in Polymeric Materials via Photochemical [2+2] Cycloaddition. Chem Mater 2004;16:3982-4.

[24] Ling J, Rong MZ, Zhang MQ. Photo-stimulated self-healing polyurethane containing dihydroxyl coumarin derivatives. Polymer 2012;53:2691-8.

[25] Froimowicz P, Frey H, Landfester K. Towards the Generation of Self-Healing Materials by Means of a Reversible Photo-induced Approach. Macromol Rapid Commun 2011;32:468-73. 
[26] Fawcett AS, Brook MA. Thermoplastic Silicone Elastomers through SelfAssociation of Pendant Coumarin Groups. Macromolecules 2014;47:1656-63.

[27] Ono T, Nobori T, Lehn J-M. Dynamic polymer blends-component recombination between neat dynamic covalent polymers at room temperature. Chem Commun 2005:1522-4.

[28] Deng G, Tang C, Li F, Jiang H, Chen Y. Covalent Cross-Linked Polymer Gels with Reversible Sol- Gel Transition and Self-Healing Properties. Macromolecules 2010;43:1191-4.

[29] Cleland WW. Dithiothreitol, a New Protective Reagent for SH Groups. Biochemistry 1964;3:480-2.

[30] Tobolsky AV, MacKnight WJ, Takahashi M. Relaxation of Disulfide and Tetrasulfide Polymers. J Phys Chem 1964;68:787-90.

[31] Adhikari B, De D, Maiti S. Reclamation and recycling of waste rubber. Prog Polym Sci 2000;25:909-48.

[32] Canadell J, Goossens H, Klumperman B. Self-Healing Materials Based on Disulfide Links. Macromolecules 2011;44:2536-41.

[33] Yoon JA, Kamada J, Koynov K, Mohin J, Nicolaÿ R, Zhang Y, Balazs AC, Kowalewski T, Matyjaszewski K. Self-Healing Polymer Films Based on ThiolDisulfide Exchange Reactions and Self-Healing Kinetics Measured Using Atomic Force Microscopy. Macromolecules 2011;45:142-9.

[34] Lafont U, van Zeijl H, van der Zwaag S. Influence of Cross-linkers on the Cohesive and Adhesive Self-Healing Ability of Polysulfide-Based Thermosets. ACS Appl Mater Interfaces 2012;4:6280-8.

[35] AbdolahZadeh M, Esteves AC, van der Zwaag S, Garcia SJ. Healable dual organic-inorganic crosslinked sol-gel based polymers: Crosslinking density and tetrasulfide content effect. J Polym Sci Part A Polym Chem 2014;52:1953-61.

[36] Zheng P, McCarthy TJ. A Surprise from 1954: Siloxane Equilibration Is a Simple, Robust, and Obvious Polymer Self-Healing Mechanism. J Am Chem Soc 2012;134:2024-7. 
[37] Ying H, Zhang Y, Cheng J. Dynamic urea bond for the design of reversible and self-healing polymers. Nat Commun 2014;5:3218/1-9.

[38] Amamoto Y, Kamada J, Otsuka H, Takahara A, Matyjaszewski K. Repeatable Photoinduced Self-Healing of Covalently Cross-Linked Polymers through Reshuffling of Trithiocarbonate Units. Angew Chem Int Ed 2011;50:1660-3.

[39] Amamoto Y, Otsuka H, Takahara A, Matyjaszewski K. Self-Healing of Covalently Cross-Linked Polymers by Reshuffling Thiuram Disulfide Moieties in Air under Visible Light. Adv Mater 2012;24:3975-80.

[40] García-Con LM, Whitcombe MJ, Piletska EV, Piletsky SA. A Sulfur-Sulfur CrossLinked Polymer Synthesized from a Polymerizable Dithiocarbamate as a Source of Dormant Radicals. Angew Chem Int Ed 2010;49:4075-8.

[41] Imato K, Nishihara M, Kanehara T, Amamoto Y, Takahara A, Otsuka H. SelfHealing of Chemical Gels Cross-Linked by Diarylbibenzofuranone-Based TriggerFree Dynamic Covalent Bonds at Room Temperature. Angew Chem Int Ed 2012;51:1138-42.

[42] Yuan CE, Rong MZ, Zhang MQ, Zhang ZP, Yuan YC. Self-Healing of Polymers via Synchronous Covalent Bond Fission/Radical Recombination. Chem Mater 2011;23:5076-81.

[43] Zhang ZP, Rong MZ, Zhang MQ, Yuan Ce. Alkoxyamine with reduced homolysis temperature and its application in repeated autonomous self-healing of stiff polymers. Polym Chem 2013;4:4648-54.

[44] Yuan Ce, Rong MZ, Zhang MQ. Self-healing polyurethane elastomer with thermally reversible alkoxyamines as crosslinkages. Polymer 2014;55:1782-91.

[45] Beyer MK. The mechanical strength of a covalent bond calculated by density functional theory. J Chem Phys 2000;112:7307-12.

[46] Ghosh B, Urban MW. Self-repairing oxetane-substituted chitosan polyurethane networks. Science 2009;323:1458-60.

[47] Ghosh B, Chellappan KV, Urban MW. UV-initiated self-healing of oxolanechitosan-polyurethane (OXO-CHI-PUR) networks. J Mater Chem 2012;22:16104-13. 
[48] Ghosh B, Chellappan KV, Urban MW. Self-healing inside a scratch of oxetanesubstituted chitosan-polyurethane (OXE-CHI-PUR) networks. J Mater Chem $2011 ; 21: 14473-86$.

[49] Yang Y, Urban MW. Self-Repairable Polyurethane Networks by Atmospheric Carbon Dioxide and Water. Angew Chem Int Ed 2014;53:12142-7.

[50] Telitel S, Amamoto Y, Poly J, Morlet-Savary F, Soppera O, Lalevee J, Matyjaszewski K. Introduction of self-healing properties into covalent polymer networks via the photodissociation of alkoxyamine junctions. Polym Chem 2014;5:921-30.

[51] Lehn JM. Supramolecular Chemistry-Scope and Perspectives Molecules, Supermolecules, and Molecular Devices (Nobel Lecture). Angew Chem Int Ed 1988;27:89-112.

[52] Cordier P, Tournilhac F, Soulié-Ziakovic C, Leibler L. Self-healing and thermoreversible rubber from supramolecular assembly. Nature 2008;451:977-80.

[53] Herbst F, Seiffert S, Binder WH. Dynamic supramolecular poly (isobutylene) s for self-healing materials. Polym Chem 2012;3:3084-92.

[54] van Gemert GML, Peeters JW, Söntjens SHM, Janssen HM, Bosman AW. SelfHealing Supramolecular Polymers In Action. Macromol Chem Phys 2012;213:234-42.

[55] Montarnal D, Tournilhac F, Hidalgo M, Couturier JL, Leibler L. Versatile one-pot synthesis of supramolecular plastics and self-healing rubbers. J Am Chem Soc 2009;131:7966-7.

[56] Zhang R, Yan T, Lechner BD, Schroter K, Liang Y, Li B, Furtado F, Sun P, Saalwächter K. Heterogeneity, Segmental and Hydrogen Bond Dynamics, and Aging of Supramolecular Self-Healing Rubber. Macromolecules 2013;46:1841-50.

[57] Maes F, Montarnal D, Cantournet S, Tournilhac F, Corté L, Leibler L. Activation and deactivation of self-healing in supramolecular rubbers. Soft Matter 2012;8:1681-7. 
[58] Hilger C, Stadler R, Liane L, Freitas dL. Multiphase thermoplastic elastomers by combination of covalent and association chain structures: 2 Small-strain dynamic mechanical properties. Polymer 1990;31:818-23.

[59] Hilger C, Stadler R. Cooperative structure formation by combination of covalent and association chain polymers: 4 Designing functional groups for supramolecular structure formation. Polymer 1991;32:3244-9.

[60] Herbst F, Schroter K, Gunkel I, Groger S, Thurn-Albrecht T, Balbach J, Binder WH. Aggregation and Chain Dynamics in Supramolecular Polymers by Dynamic Rheology: Cluster Formation and Self-Aggregation. Macromolecules 2010;43:10006-16.

[61] Beijer FH, Sijbesma RP, Kooijman H, Spek AL, Meijer E. Strong dimerization of ureidopyrimidones via quadruple hydrogen bonding. J Am Chem Soc 1998;120:6761-9.

[62] Söntjens SH, Sijbesma RP, van Genderen MH, Meijer E. Stability and lifetime of quadruply hydrogen bonded 2-ureido-4 [1 H]-pyrimidinone dimers. J Am Chem Soc 2000;122:7487-93.

[63] Feldman KE, Kade MJ, de Greef TF, Meijer E, Kramer EJ, Hawker CJ. Polymers with multiple hydrogen-bonded end groups and their blends. Macromolecules 2008;41:4694-700.

[64] Yamauchi K, Lizotte JR, Hercules DM, Vergne MJ, Long TE. Combinations of Microphase Separation and Terminal Multiple Hydrogen Bonding in Novel Macromolecules. J Am Chem Soc 2002;124:8599-604.

[65] Yamauchi K, Lizotte JR, Long TE. Thermoreversible Poly(alkyl acrylates) Consisting of Self-Complementary Multiple Hydrogen Bonding. Macromolecules 2003;36:1083-8.

[66] Cui J, del Campo A. Multivalent H-bonds for self-healing hydrogels. Chem Commun 2012;48:9302-4.

[67] Hentschel J, Kushner AM, Ziller J, Guan Z. Self-Healing Supramolecular Block Copolymers. Angew Chem Int Ed 2012;51:10561-5. 
[68] Chen Y, Guan Z. Multivalent hydrogen bonding block copolymers self-assemble into strong and tough self-healing materials. Chem Commun 2014;50:10868-70.

[69] Manners I. Synthetic metal-containing polymers. New York: John Wiley \& Sons Inc, 2004. 275 pp.

[70] Whittell GR, Hager MD, Schubert US, Manners I. Functional soft materials from metallopolymers and metallosupramolecular polymers. Nat Mater 2011;10:176-88.

[71] Hardy CG, Zhang J, Yan Y, Ren L, Tang C. Metallopolymers with transition metals in the side-chain by living and controlled polymerization techniques. Prog Polym Sci 2014;39:1742-96.

[72] Williams KA, Boydston AJ, Bielawski CW. Towards electrically conductive, selfhealing materials. J R Soc Interface 2007;4:359-62.

[73] Burnworth M, Tang L, Kumpfer JR, Duncan AJ, Beyer FL, Fiore GL, Rowan SJ, Weder C. Optically healable supramolecular polymers. Nature 2011;472:334-7.

[74] Bode S, Zedler L, Schacher FH, Dietzek B, Schmitt M, Popp J, Hager M, Schubert US. Self-Healing Polymer Coatings Based on Crosslinked Metallosupramolecular Copolymers. Adv Mater 2013;25:1634-8.

[75] Holten-Andersen N, Harrington MJ, Birkedal H, Lee BP, Messersmith PB, Lee KYC, Waite JH. pH-induced metal-ligand cross-links inspired by mussel yield self-healing polymer networks with near-covalent elastic moduli. Proc Natl Acad Sci USA 2011;108:2651-5.

[76] Ceylan H, Urel M, Erkal TS, Tekinay AB, Dana A, Guler MO. Mussel Inspired Dynamic Cross-Linking of Self-Healing Peptide Nanofiber Network. Adv Funct Mater 2013;23:2081-90.

[77] Wang Z, Urban MW. Facile UV-healable polyethylenimine-copper (C 2 H 5 N$\mathrm{Cu}$ ) supramolecular polymer networks. Polym Chem 2013;4:4897-901.

[78] Gohy JF, Lohmeijer BG, Schubert US. Reversible Metallo-Supramolecular Block Copolymer Micelles Containing a Soft Core. Macromol Rapid Commun 2002;23:555-60.

[79] Wild A, Winter A, Schlütter F, Schubert US. Advances in the field of $\pi$-conjugated 2, 2': 6', 2"-terpyridines. Chem Soc Rev 2011;40:1459-511. 
[80] Constable EC. 2, 2': 6', 2"-Terpyridines: From chemical obscurity to common supramolecular motifs. Chem Soc Rev 2007;36:246-53.

[81] Vaccaro E, Waite JH. Yield and post-yield behavior of mussel byssal thread: a self-healing biomolecular material. Biomacromolecules 2001;2:906-11.

[82] Lee BP, Dalsin JL, Messersmith PB. Synthesis and gelation of DOPA-modified poly (ethylene glycol) hydrogels. Biomacromolecules 2002;3:1038-47.

[83] Krogsgaard M, Behrens MA, Pedersen JS, Birkedal H. Self-healing musselinspired multi-pH-responsive hydrogels. Biomacromolecules 2013;14:297-301.

[84] He L, Fullenkamp DE, Rivera JG, Messersmith PB. pH responsive self-healing hydrogels formed by boronate-catechol complexation. Chem Commun 2011;47:7497-9.

[85] Vatankhah-Varnoosfaderani M, Hashmi S, GhavamiNejad A, Stadler FJ. Rapid self-healing and triple stimuli responsiveness of a supramolecular polymer gel based on boron-catechol interactions in a novel water-soluble mussel-inspired copolymer. Polym Chem 2014;5:512-23.

[86] Kersey FR, Loveless DM, Craig SL. A hybrid polymer gel with controlled rates of cross-link rupture and self-repair. J R Soc Interface 2007;4:373-80.

[87] Wang Z, Yang Y, Burtovyy R, Luzinov I, Urban MW. UV-induced self-repairing polydimethylsiloxane-polyurethane (PDMS-PUR) and polyethylene glycolpolyurethane (PEG-PUR) Cu-catalyzed networks. J Mater Chem A 2014;2:1552734.

[88] Burattini S, Colquhoun HM, Greenland BW, Hayes W. A novel self-healing supramolecular polymer system. Faraday Discuss 2009;143:251-64.

[89] Burattini S, Colquhoun HM, Fox JD, Friedmann D, Greenland BW, Harris PJF, Hayes W, Mackay ME, Rowan SJ. A self-repairing, supramolecular polymer system: healability as a consequence of donor-acceptor $[\pi]-[\pi]$ stacking interactions. Chem Commun 2009:6717-9.

[90] Burattini S, Greenland BW, Merino DH, Weng W, Seppala J, Colquhoun HM, Hayes W, Mackay ME, Hamley IW, Rowan SJ. A healable supramolecular 
polymer blend based on aromatic $\pi-\pi$ stacking and hydrogen-bonding interactions. J Am Chem Soc 2010;132:12051-8.

[91] Burattini S, Greenland BW, Hayes W, Mackay ME, Rowan SJ, Colquhoun HM. A Supramolecular Polymer Based on Tweezer-Type $\pi-\pi$ Stacking Interactions: Molecular Design for Healability and Enhanced Toughness. Chem Mater 2010;23:6-8.

[92] Xu Z, Peng J, Yan N, Yu H, Zhang S, Liu K, Fang Y. Simple design but marvelous performances: molecular gels of superior strength and self-healing properties. Soft Matter 2013;9:1091-9.

[93] Eisenberg A, Hird B, Moore R. A new multiplet-cluster model for the morphology of random ionomers. Macromolecules 1990;23:4098-107.

[94] Kalista SJ, Stephen J. Self-healing of thermoplastic poly (ethylene-co-methacrylic acid) copolymers following projectile puncture. M.S. Thesis; Virginia Polytechnic Institute and State University, Blacksburg VA, 2003. 67 pp.

[95] Kalista SJ, Ward TC. Thermal characteristics of the self-healing response in poly (ethylene-co-methacrylic acid) copolymers. J R Soc Interface 2007;4:405-11.

[96] Varley RJ, Shen S, van der Zwaag S. The effect of cluster plasticisation on the self- healing behaviour of ionomers. Polymer 2010;51:679-86.

[97] Reisch A, Roger E, Phoeung T, Antheaume C, Orthlieb C, Boulmedais F, Lavalle P, Schlenoff JB, Frisch B, Schaaf P. On the Benefits of Rubbing Salt in the Cut: Self-Healing of Saloplastic PAA/PAH Compact Polyelectrolyte Complexes. Adv Mater 2014;26:2547-51.

[98] Kalista SJ, Pflug JR, Varley RJ. Effect of ionic content on ballistic self-healing in EMAA copolymers and ionomers. Polym Chem 2013;4:4910-26.

[99] South AB, Lyon LA. Autonomic Self-Healing of Hydrogel Thin Films. Angew Chem Int Ed 2009;49:767-71.

[100] Serpe MJ, Jones CD, Lyon LA. Layer-by-layer deposition of thermoresponsive microgel thin films. Langmuir 2003;19:8759-64.

[101] Wang X, Liu F, Zheng X, Sun J. Water-Enabled Self-Healing of Polyelectrolyte Multilayer Coatings. Angew Chem Int Ed 2011;50:11378-81. 
[102] Huang Y, Lawrence PG, Lapitsky Y. Self-Assembly of Stiff, Adhesive and SelfHealing Gels from Common Polyelectrolytes. Langmuir 2014;30:7771-7.

[103] Ahn BK, Lee DW, Israelachvili JN, Waite JH. Surface-initiated self-healing of polymers in aqueous media. Nat Mater 2014;13:867-72.

[104] Szejtli J. Introduction and general overview of cyclodextrin chemistry. Chem Rev 1998;98:1743-54.

[105] Pluth MD, Raymond KN. Reversible guest exchange mechanisms in supramolecular host-guest assemblies. Chem Soc Rev 2007;36:161-71.

[106] Nakahata M, Takashima Y, Yamaguchi H, Harada A. Redox-responsive selfhealing materials formed from host-guest polymers. Nat Commun 2011;2:511/1-6.

[107] Kakuta T, Takashima Y, Nakahata M, Otsubo M, Yamaguchi H, Harada A. Preorganized Hydrogel: Self-Healing Properties of Supramolecular Hydrogels Formed by Polymerization of Host-Guest Monomers that Contain Cyclodextrins and Hydrophobic Guest Groups. Adv Mater 2013;25:2849-53.

[108] Appel EA, Biedermann F, Rauwald U, Jones ST, Zayed JM, Scherman OA. Supramolecular Cross-Linked Networks via Host-Guest Complexation with Cucurbit [8] uril. J Am Chem Soc 2010;132:14251-60.

[109] Stayton PS, Shimoboji T, Long C, Chilkoti A, Ghen G, Harris JM, Hoffman AS. Control of protein-ligand recognition using a stimuli-responsive polymer. Nature 1995;378:472-4.

[110] Petka WA, Harden JL, McGrath KP, Wirtz D, Tirrell DA. Reversible Hydrogels from Self-Assembling Artificial Proteins. Science 1998;281:389-92.

[111] Fogleman EA, Yount WC, Xu J, Craig SL. Modular, Well-Behaved Reversible Polymers from DNA-Based Monomers. Angew Chem Int Ed 2002;41:4026-8.

[112] Lin DC, Yurke B, Langrana NA. Mechanical properties of a reversible, DNAcrosslinked polyacrylamide hydrogel. J Biomech Eng 2004;126:104-10.

[113] Seelig G, Yurke B, Winfree E. Catalyzed Relaxation of a Metastable DNA Fuel. J Am Chem Soc 2006;128:12211-20. 
[114] Liedl T, Dietz H, Yurke B, Simmel F. Controlled Trapping and Release of Quantum Dots in a DNA-Switchable Hydrogel. Small 2007;3:1688-93.

[115] Zhang DY, Turberfield AJ, Yurke B, Winfree E. Engineering entropy-driven reactions and networks catalyzed by DNA. Science 2007;318:1121-5.

[116] Yoonessi M, Shi Y, Scheiman DA, Lebron-Colon M, Tigelaar DM, Weiss R, Meador MA. Graphene polyimide nanocomposites; thermal, mechanical, and hightemperature shape memory effects. ACS Nano 2012;6:7644-55.

[117] Wang C, Liu N, Allen R, Tok JBH, Wu Y, Zhang F, Chen Y, Bao Z. A Rapid and Efficient Self-Healing Thermo-Reversible Elastomer Crosslinked with Graphene Oxide. Adv Mater 2013;25:5785-90.

[118] Yadav SK, Cho JW. Functionalized graphene nanoplatelets for enhanced mechanical and thermal properties of polyurethane nanocomposites. Appl Surf Sci 2013;266:360-7.

[119] Biyani MV, Foster EJ, Weder C. Light-Healable Supramolecular Nanocomposites Based on Modified Cellulose Nanocrystals. ACS Macro Lett 2013;2:236-40.

[120] Artel V, Cohen R, Aped I, Ronen M, Gerber D, Sukenik CN. Controlled formation of thiol and disulfide interfaces. Langmuir 2012;29:191-8.

[121] Engel T, Kickelbick G. Self-healing nanocomposites from silica-polymer coreshell nanoparticles. Polym Int 2014;63:915-23.

[122] Froimowicz P, Klinger D, Landfester K. Photoreactive Nanoparticles as Nanometric Building Blocks for the Generation of Self-Healing Hydrogel Thin Films. Chem Eur J 2011;17:12465-75.

[123] Smith AR, Watson DF. Photochemically triggered assembly of composite nanomaterials through the photodimerization of adsorbed anthracene derivatives. Chem Mater 2009;22:294-304.

[124] Vaiyapuri R, Greenland BW, Rowan SJ, Colquhoun HM, Elliott JM, Hayes W. Thermoresponsive supramolecular polymer network comprising pyrenefunctionalized gold nanoparticles and a chain-folding polydiimide. Macromolecules 2012;45:5567-74. 
[125] Cheng G, Vautravers NR, Morris RE, Cole-Hamilton DJ. Synthesis of functional cubes from octavinylsilsesquioxane (OVS). Org Biomol Chem 2008;6:4662-7.

[126] Liang K, Li G, Toghiani H, Koo JH, Pittman CU. Cyanate ester/polyhedral oligomeric silsesquioxane (POSS) nanocomposites: synthesis and characterization. Chem Mater 2006;18:301-12.

[127] Xue Y, Liu Y, Lu F, Qu J, Chen H, Dai L. Functionalization of graphene oxide with polyhedral oligomeric silsesquioxane (POSS) for multifunctional applications. J Phys Chem Lett 2012;3:1607-12.

[128] Xu Z, Zhao Y, Wang X, Lin T. A thermally healable polyhedral oligomeric silsesquioxane (POSS) nanocomposite based on Diels-Alder chemistry. Chem Commun 2013;49:6755-7.

[129] Kango S, Kalia S, Celli A, Njuguna J, Habibi Y, Kumar R. Surface modification of inorganic nanoparticles for development of organic-inorganic nanocomposites - A review. Prog Polym Sci 2013;38:1232-61.

[130] Lee JY, Zhang Q, Emrick T, Crosby AJ. Nanoparticle alignment and repulsion during failure of glassy polymer nanocomposites. Macromolecules 2006;39:73926.

[131] Vaiyapuri R, Greenland BW, Colquhoun HM, Elliott JM, Hayes W. Molecular recognition between functionalized gold nanoparticles and healable, supramolecular polymer blends-a route to property enhancement. Polym Chem 2013;4:4902-9.

[132] Corten CC, Urban MW. Repairing polymers using oscillating magnetic field. Adv Mater 2009;21:5011-5.

[133] Alessandri I. Writing, Self-Healing, and Self-Erasing on Conductive PressureSensitive Adhesives. Small 2010;6:1679-85.

[134] Cortie MB, McDonagh AM. Synthesis and optical properties of hybrid and alloy plasmonic nanoparticles. Chem Rev 2011;111:3713-35.

[135] Giannini V, Fernandez-Dominguez AI, Heck SC, Maier SA. Plasmonic nanoantennas: fundamentals and their use in controlling the radiative properties of nanoemitters. Chem Rev 2011;111:3888-912. 
[136] Amendola V, Dini D, Polizzi S, Shen J, Kadish KM, Calvete MJF, Hanack M, Meneghetti M. Self-healing of gold nanoparticles in the presence of zinc phthalocyanines and their very efficient nonlinear absorption performances. J Phys Chem C 2009;113:8688-95.

[137] Amendola V, Meneghetti M. Advances in self-healing optical materials. J Mater Chem 2012;22:24501-8.

[138] Fiore GL, Rowan SJ, Weder C. Optically healable polymers. Chem Soc Rev 2013;42:7278-88.

[139] Zhang H, Xia H, Zhao Y. Optically triggered and spatially controllable shapememory polymer-gold nanoparticle composite materials. J Mater Chem 2012;22:845-9.

[140] Fox J, Wie JJ, Greenland BW, Burattini S, Hayes W, Colquhoun HM, Mackay ME, Rowan JR. High-Strength, Healable, Supramolecular Polymer Nanocomposites. J Am Chem Soc 2012;134:5362-8.

[141] Chen H, Shao L, Li Q, Wang J. Gold nanorods and their plasmonic properties. Chem Soc Rev 2013;42:2679-724.

[142] Pearce ME, Melanko JB, Salem AK. Multifunctional nanorods for biomedical applications. Pharm Res 2007;24:2335-52.

[143] Kim J, Jung IS, Kim SY, Lee E, Kang JK, Sakamoto S, Yamaguchi K, Kim K. New Cucurbituril Homologues: -Syntheses, Isolation, Characterization, and X-ray Crystal Structures of Cucurbit[n]uril ( $n=5,7$, and 8). J Am Chem Soc 2000;122:540-1.

[144] McKee JR, Appel EA, Seitsonen J, Kontturi E, Scherman OA, Ikkala O. Healable, Stable and Stiff Hydrogels: Combining Conflicting Properties Using Dynamic and Selective Three-Component Recognition with Reinforcing Cellulose Nanorods. Adv Funct Mater 2014;24:2706-13.

[145] Novoselov KS, Geim AK, Morozov S, Jiang D, Zhang Y, Dubonos S, Grigorieva IV, Firsov AA. Electric field effect in atomically thin carbon films. Science 2004;306:666-9. 
[146] Stankovich S, Dikin DA, Dommett GHB, Kohlhaas KM, Zimney EJ, Stach EA, Piner RD, Nguyen ST, Ruoff RS. Graphene-based composite materials. Nature 2006;442:282-6.

[147] Kohlmeyer RR, Lor M, Chen J. Remote, local, and chemical programming of healable multishape memory polymer nanocomposites. Nano Lett 2012;12:275762.

[148] Huang L, Yi N, Wu Y, Zhang Y, Zhang Q, Huang Y, Ma Y, Chen Y. Multichannel and Repeatable Self-Healing of Mechanical Enhanced Graphene-Thermoplastic Polyurethane Composites. Adv Mater 2013;25:2224-8.

[149] Kim JT, Kim BK, Kim EY, Kwon SH, Jeong HM. Synthesis and properties of near IR induced self-healable polyurethane/graphene nanocomposites. Eur Polym J 2013;49:3889-96.

[150] Sun H, You X, Jiang Y, Guan G, Fang X, Deng J, Chen P, Luo Y, Peng H. SelfHealable Electrically Conducting Wires for Wearable Microelectronics. Angew Chem Int Ed 2014;53:9526-31.

[151] Gong C, Liang J, Hu W, Niu X, Ma S, Hahn HT, Pei Q. A Healable, Semitransparent Silver Nanowire-Polymer Composite Conductor. Adv Mater 2013;25:4186-91.

[152] Peterson AM, Jensen RE, Palmese GR. Thermoreversible and remendable glasspolymer interface for fiber-reinforced composites. Compos Sci Technol 2011;71:586-92.

[153] Jones AR, Blaiszik BJ, White SR, Sottos NR. Full recovery of fiber/matrix interfacial bond strength using a microencapsulated solvent-based healing system. Compos Sci Technol 2013;79:1-7.

[154] Wool RP. Crack healing in semicrystalline polymers, block copolymers and filled elastomers. Adhesion and Adsorption of Polymers. Polym Sci Technol 1980;12A:341-62.

[155] Fall R. Puncture reversal of ethylene ionomers-mechanistic studies. M.S. Thesis; Blacksburg VA: Virginia Tech 2001. 65 pp. 
[156] Kalista Jr SJ, Ward TC, Oyetunji Z. Self-healing of poly (ethylene-co-methacrylic acid) copolymers following projectile puncture. Mech Adv Mater Struc 2007;14:391-7.

[157] Wool R, O'connor K. A theory crack healing in polymers. J Appl Phys 1981;52:5953-63.

[158] de Gennes PG. Reptation of a polymer chain in the presence of fixed obstacles. J Chem Phys 1971;55:572-9.

[159] Lin C, Lee S, Liu K. Methanol-Induced crack healing in poly (methyl methacrylate). Poly Eng Sci 1990;30:1399-406.

[160] Jud K, Kausch H, Williams J. Fracture mechanics studies of crack healing and welding of polymers. J Mater Sci 1981;16:204-10.

[161] Prager S, Tirrell M. The healing process at polymer-polymer interfaces. J Chem Phys 1981;75:5194-8.

[162] Forrest J, Dalnoki-Veress K, Stevens J, Dutcher J. Effect of free surfaces on the glass transition temperature of thin polymer films. Phys Rev Lett 1996;77:2002-5.

[163] Forrest J, Dalnoki-Veress K, Dutcher J. Interface and chain confinement effects on the glass transition temperature of thin polymer films. Phys Rev E 1997;56:570516.

[164] Richard A. Interface and surface effects on the glass-transition temperature in thin polymer films. Faraday Discuss 1994;98:219-30.

[165] Wool RP. Twinkling fractal theory of the glass transition. J Polym Sci Part B Polym Phys 2008;46:2765-78. 
Figure Captions

Figure 1. A - Schematic illustration of a hybrid material consisting of soft and hard domains that facilitate rearrangements of the two domains.

B - Length scale of heterogeneities in self-healing materials.

Figure 2. A- Structure of the dendritic building block containing anthracene groups on the periphery;

B - Self-repair process of polymer films synthesized from the dendritic building upon crosslinking via anthracene cycloaddition [25], Copyright 2011. Reproduced with permission from John Wiley \& Sons Inc.

Figure 3. Self-repairing exchange reactions responsible for self-healing. [27], [32], [36], [37].

Figure 4. Self-healing polymers containing S-S groups that exhibit structural heterogeneities: A - Formation of star polymers bearing S-S bonds on the periphery [33], Copyright 2011. Adapted with permission from the American Chemical Society; B - Polymer network composed of silicone alkoxides and epoxy resin incorporated with reversible tetrasulfide bonds [35], Copyright 2014. Adapted with permission from John Wiley \& Sons Inc.

Figure 5. Stable free radical mediated reshuffle reactions responsible for self-repair: $\mathrm{A}$ - trithiocarbonates reshuffling [38]; $\mathrm{B}$ - thiuram disulfide [39]; $\mathrm{C}$ diarylbibenzofuranone [41]; D - alkoxyamine [42].

Figure 6. Self-repair of polyurethane networks:

A - oxetane-substituted chitosan polyurethane (OXE-CHI-PUR) network; A' - Atomic force microscopy (AFM) images of undamaged (A'1), damaged (A'2), UV-exposed (A'2 ${ }^{1}, \mathrm{~A}^{\prime} 2^{2}$ ) and repaired (A'3) OXE-CHIPUR, and a plot of $\mathrm{d}(\Delta l) / \mathrm{dT}$ vs. temperature of undamaged, damaged, and repaired networks $[46,48]$;

B - Methyl $\alpha$-D-glucopyranoside modified polyurethane (MGP-PUR) network; B' - Optical images of MGP-PUR self-repair in the presence of $\mathrm{CO}_{2}$ and $\mathrm{H}_{2} \mathrm{O}$. The Table B' lists storage modulus (E') of MGP-PUR inside 
newly created scratch and outside of the scratch measured as a function of time at the oscillation depth of $\sim 200 \mathrm{~nm}$ [49].

Figure 7. Reversible H-bond formations responsible for self-repair and the networks' physical and self-repair properties:

A - diacids and triacids associations from renewable resources [52] ;

B - triple-H-bonds formation between thymine (Thy) and 2,6-

diaminotriaine (DAT) [53], Copyright 2012. Reproduced with permission from the Nature Group;

$\mathrm{C}$ - dimerization of ureidopyrimidinone (Upy) units by quadruple-H-bonds [54];

D - copolymers combining hard polystyrene (PS) backbones with soft polyacrylate amide (PA-A) pendant groups carrying multiple H-bonding sites, and TEM image showing heterogeneity of the polymer [13], Copyright 2012. Reproduced with permission from the Nature Group.

Figure 8. Reversible metal-ligand coordination reactions responsible for self-repair and the networks' physical and self-healing properties:

A - dynamic equilibrium of N-heterocyclic carbenes and transition metals monomeric species and their polymer [72];

B - self-repairing supramolecular network containing 2,6-bis(1'methylbenzimidazolyl)pyridine (Mebip) ligands coordinated with $\mathrm{Zn}(\mathrm{NTf})_{2}$; Poly(ethylene-co-butylene) is utilized as the main chain, resulting in phase separated lamella structure as shown in the TEM image [73], Copyright 2011. Reproduced with permission from the Nature Group;

$\mathrm{C}$ - terpyridine containing alkyl methacrylates coordinated with (iron) $\mathrm{Fe}$ (II) sulfate [74];

D - Reversible tris-catechol- $\mathrm{Fe}^{3+}$ complex [75], SEM images demonstrate self-assembled peptide nanofibers inter-crosslinked by catechol- $\mathrm{Fe}^{3+}$ complex [76];

E - polyethylenimine-copper $\left(\mathrm{C}_{2} \mathrm{H}_{5} \mathrm{~N}-\mathrm{Cu}\right)$ supramolecular polymer networks and the UV induced square planer-to-tetrahedral conformational 
changes [77], Copyright 2013. Reproduced with permission from the Royal Society of Chemistry.

Figure 9. A - Chemical structure of $\mathbf{1}$ - chain folding units containing naphthalenediimide receptor, $\mathbf{2}$ - polyamide end-capped with pyrenyl groups, and $\mathbf{3}$ polyamide end-capped with tweeter type bis-pyrenyl groups;

B - Energy-minimized models (molecular mechanics with chargeequilibration) for complexes formed between compound 1 and $\mathbf{2}([\mathbf{1}+\mathbf{2}])$, and between compound $\mathbf{1}$ and $\mathbf{3}([\mathbf{1}+\mathbf{3}])$, in which formations of two or four face-to-face $\pi$ - $\pi$ stacking interactions are shown;

C - The table illustrates the results of the tensile measurements and selfhealing conditions for blends [1+2] and [1+3] [91], Copyright 2010 . Adapted with permission from the American Chemical Society.

Figure 10. Examples of ionomers utilized in self-healing:

A - polyethylene-co-methacrylic acid) (EMMA) [94];

B - Poly(acrylic acid) (PAA)/poly(allylamine hydrochloride) (PAH) forming compact polyelectrolyte complexes (CoPECs) by ultracentrifuge [97];

C - Poly(allylamine hydrochloride) (PAH)/tripolyphosphate (TPP) ionic gel [102].

Figure 11. A - Nanomaterials; B. Selected surface modifications for nanomaterials; C

- Polymer matrix functional groups; D - Covalent/dynamic bonding formation between nanomaterials and a polymer matrix; E - Schematic illustration of dynamic interfacial rebonding in nanocomposites; driven by the partial dissociation of interfacial bonging under shear forces, followed by reforming cleaved interactions.

Figure 12. Schematic illustration of a thermally healable POSS composite based on DA reactions:

A - chemical reactions leading to composite formation;

B - self-repairing process facilitated by retro-DA reactions [128], 
Copyright 2013. Adapted with permission from the Royal Society of Chemistry.

Figure 13. Schematic illustration of a thermal reversible supramolecular composite network based on $\pi-\pi$ stacking interactions between pyrene-functionalized gold nanoparticles (AuNPs), pyrene end-capped polyamide (a) and polydiimide (b) [131], Copyright 2013. Adapted with permission from the Royal Society of Chemistry.

Figure 14. Illustration of the repairing process of magnetic nanoparticle incorporated thermo-plastic polymers facilitated by nanoparticle oscillatory movements in the presence of oscillating magnetic field [132], Copyright 2009.

Adapted with permission from John Wiley \& Sons Inc.

Figure 15. Schematic illustration of A - CNC functionalized with UPy motif, and B light triggered self-healing process of CNC-UPy/UPy-Polymer composite [119], Copyright 2013. Adapted with permission from the American Chemical Society.

Figure 16. Schematic representation of preparing highly specific, dynamic and stiff supramolecular composite hydrogels based on functionalized cellulose nanocrystals $(\mathrm{CNC})$ and $\mathrm{CB}[8]$ three-component recognition host-guest chemistry:

A - Poly(vinyl alcohol) (PVA) functionalized with the first-guest methyl viologen (PVA-MV);

B - CNCs grafted with copolymer containing protonated dimethylaminoethl methacrylate (DMAEMA) and second-guest naphthyl methacrylate (NpMA) (CNC-g-P(DMAEMA-r-NpMA); $\mathrm{C}-\mathrm{CB}[8]$ as the host motif; D - Supramolecular composite formation based on host-guest cross-linking chemistry. [144], Copyright 2014. Adapted with permission from John Wiley \& Sons Inc.

Figure 17. Schematic illustration of two types of physical processes involved in selfhealing: A - the presence of shape memory components, where elastic 
forces of spring-like polymers force the closure of damage; B - an interfacial flow causes damages repair. 

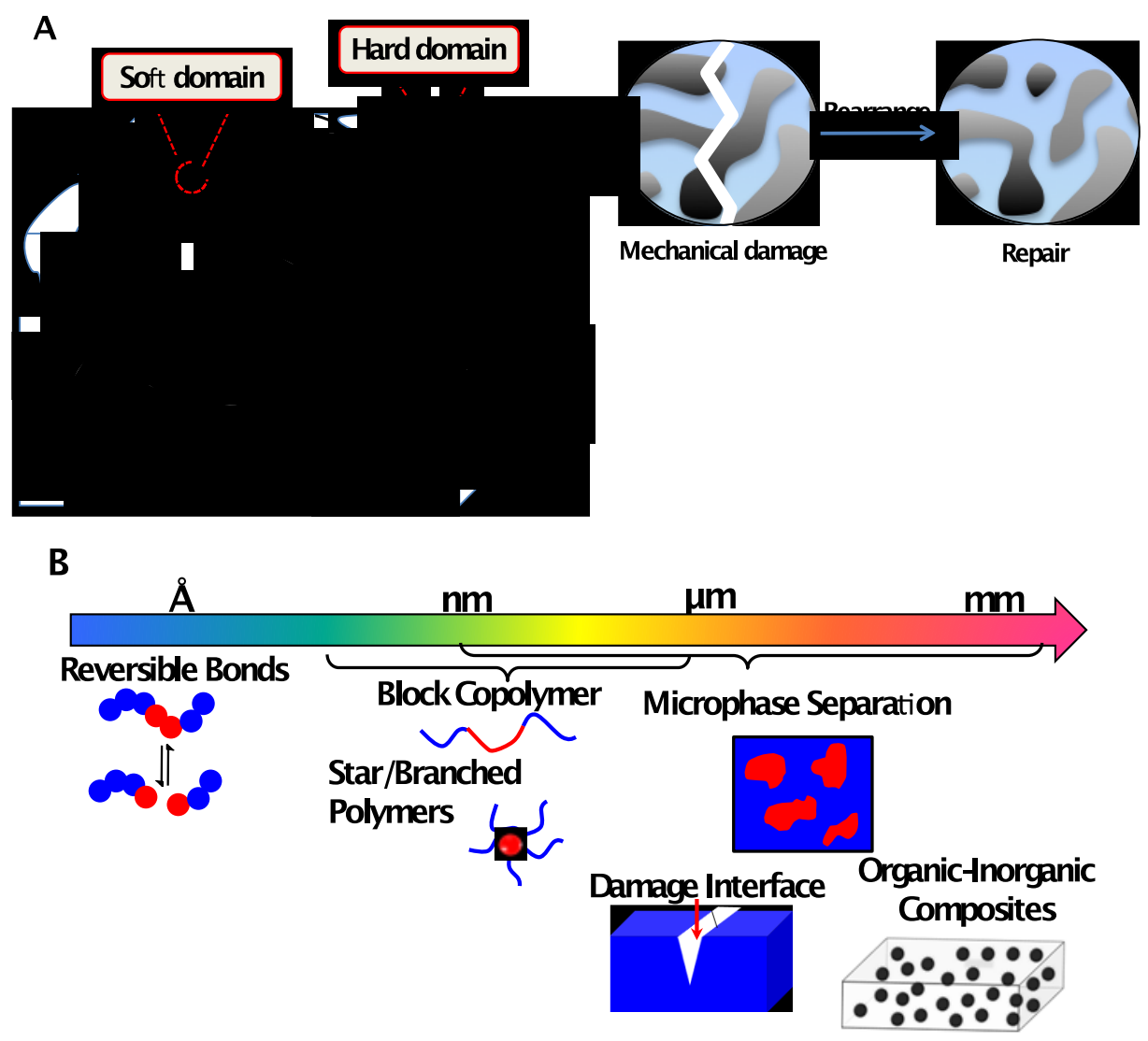

Figure 1. 


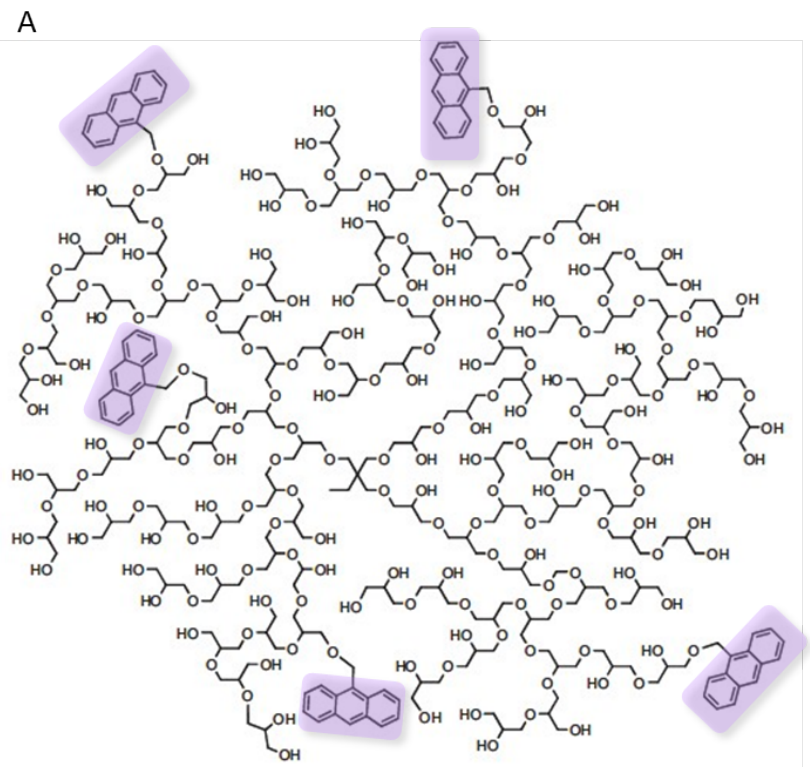

B

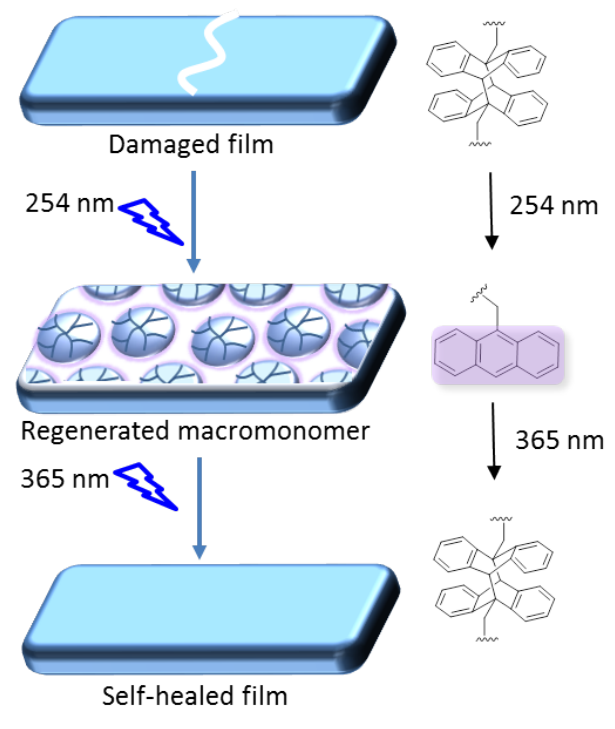

Figure 2. 


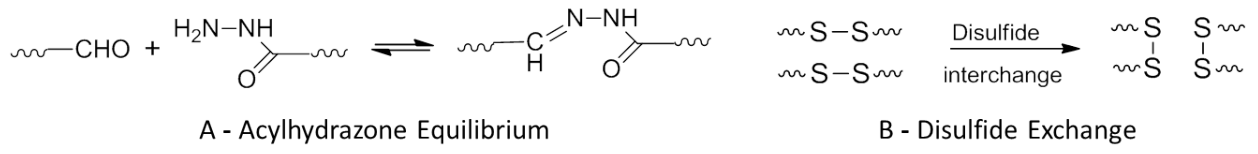

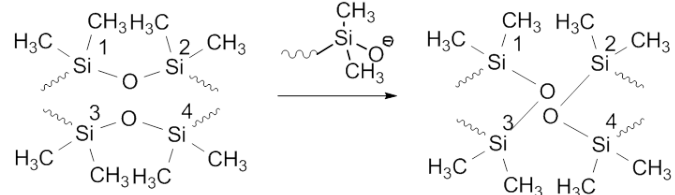

C - Siloxane Exchange

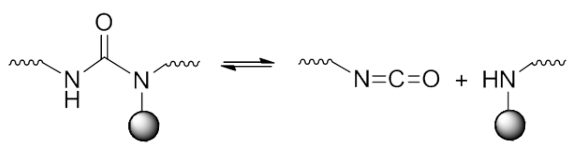

D - Reversible urea bonds with bulky substituents

Figure 3. 


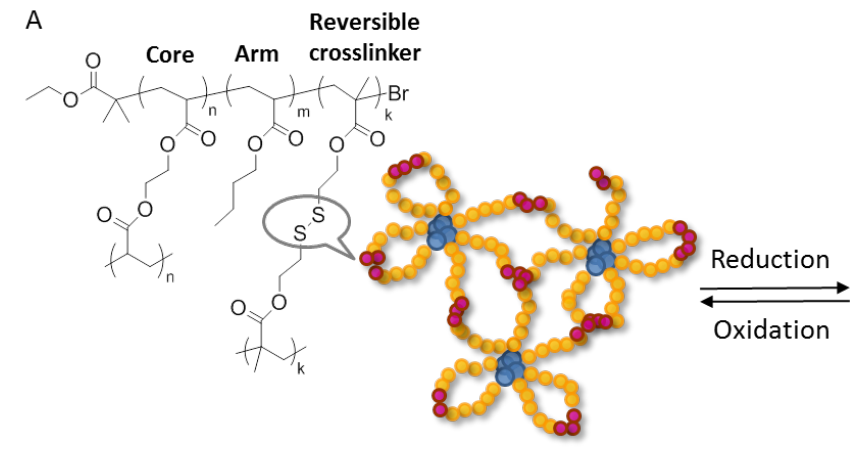

Reversibly crosslinked star polymer gel

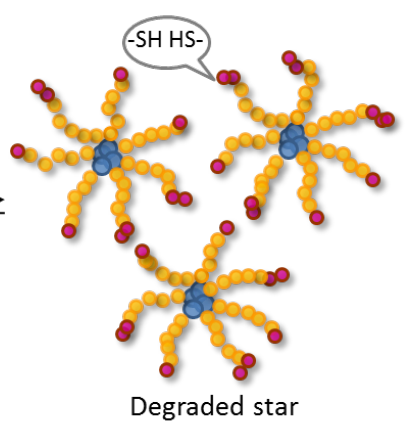

Degraded star

B

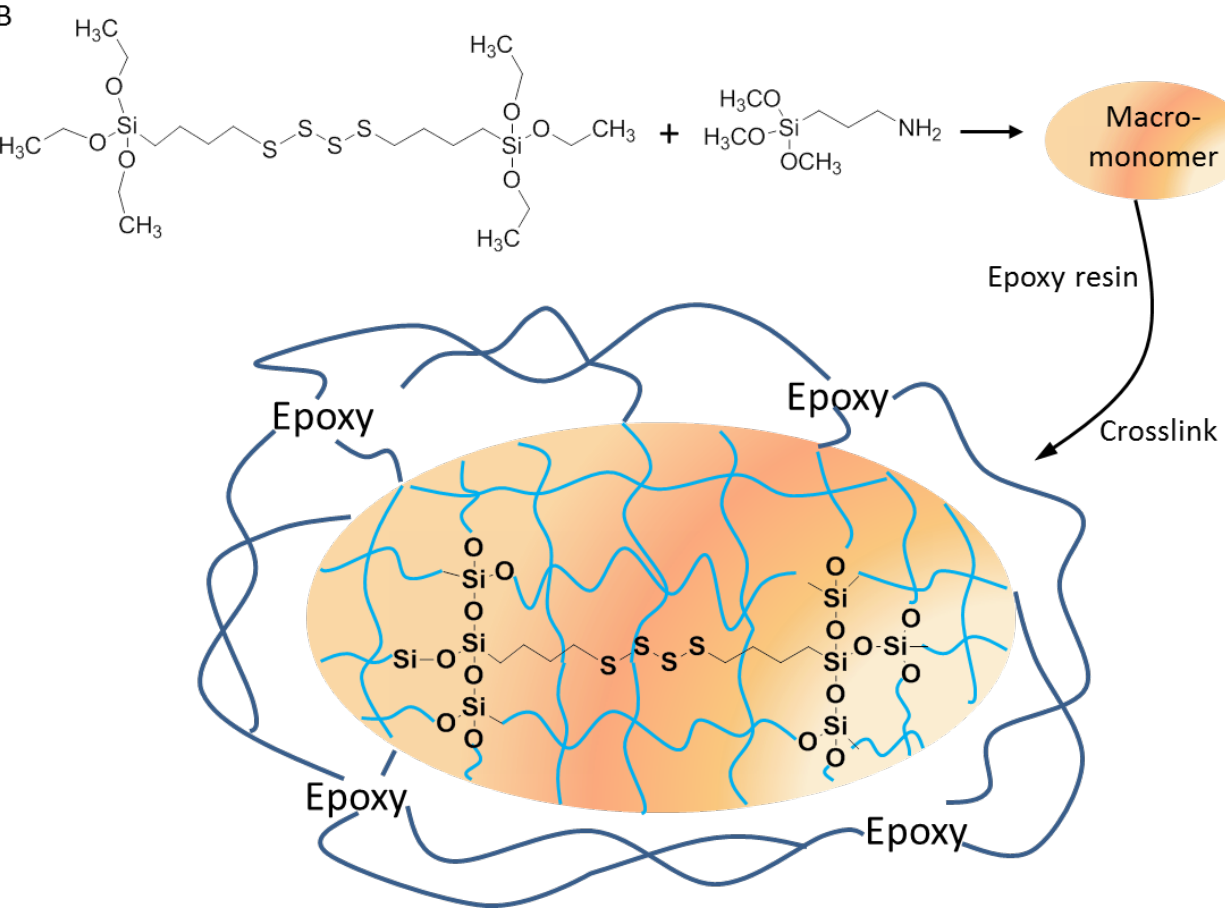

Figure 4. 

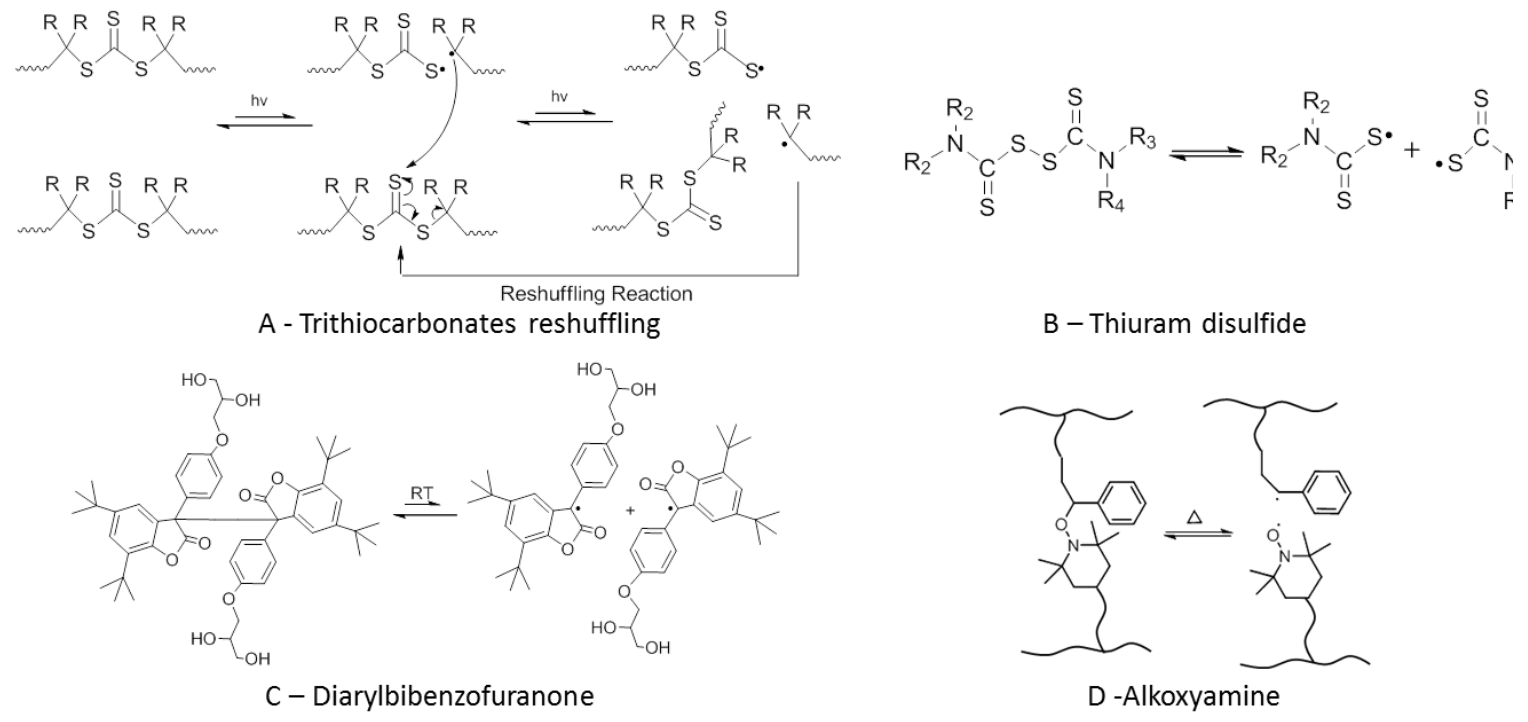

B - Thiuram disulfide

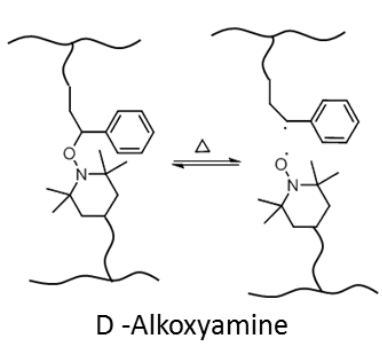

Figure 5. 

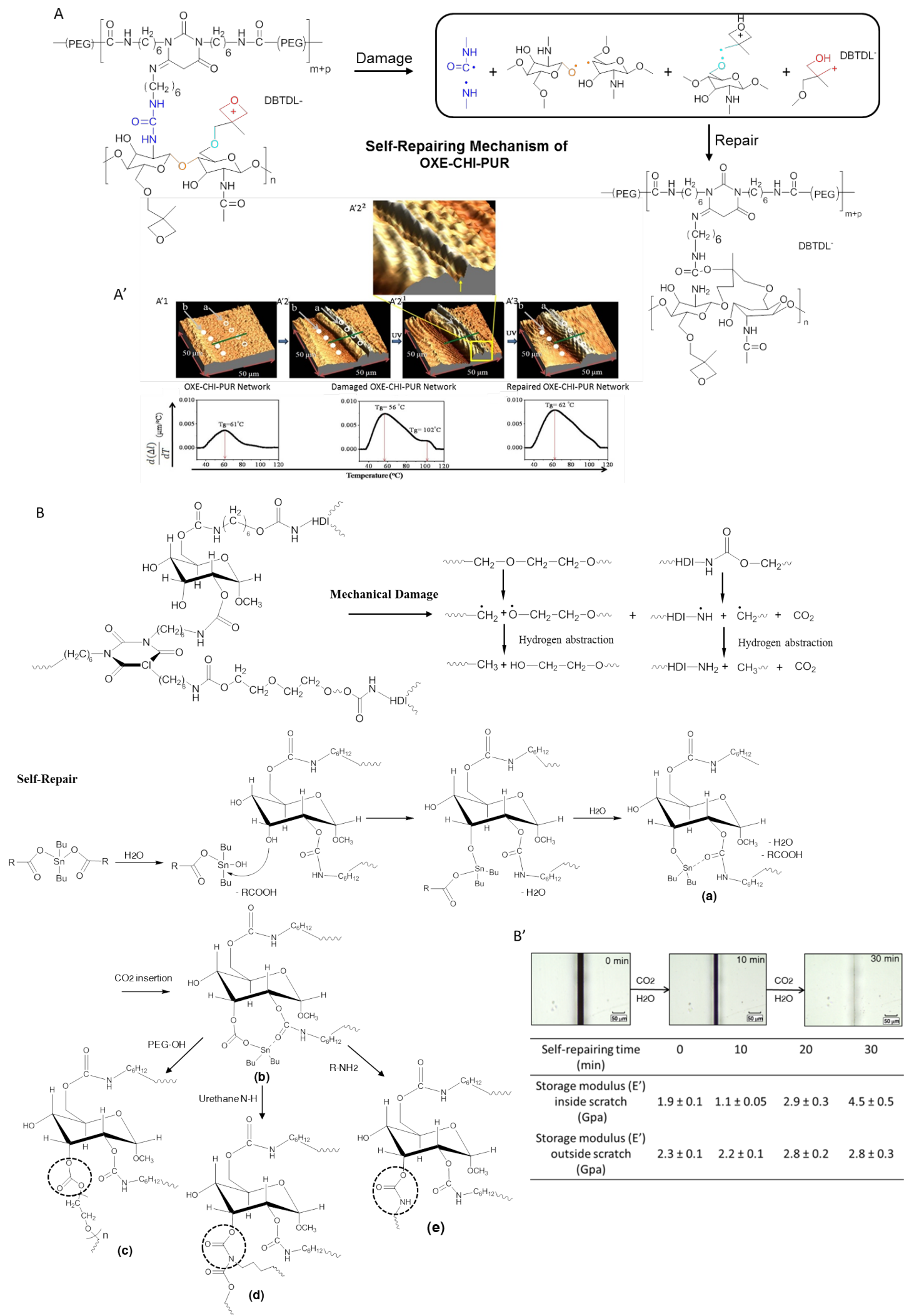

Figure 6. 


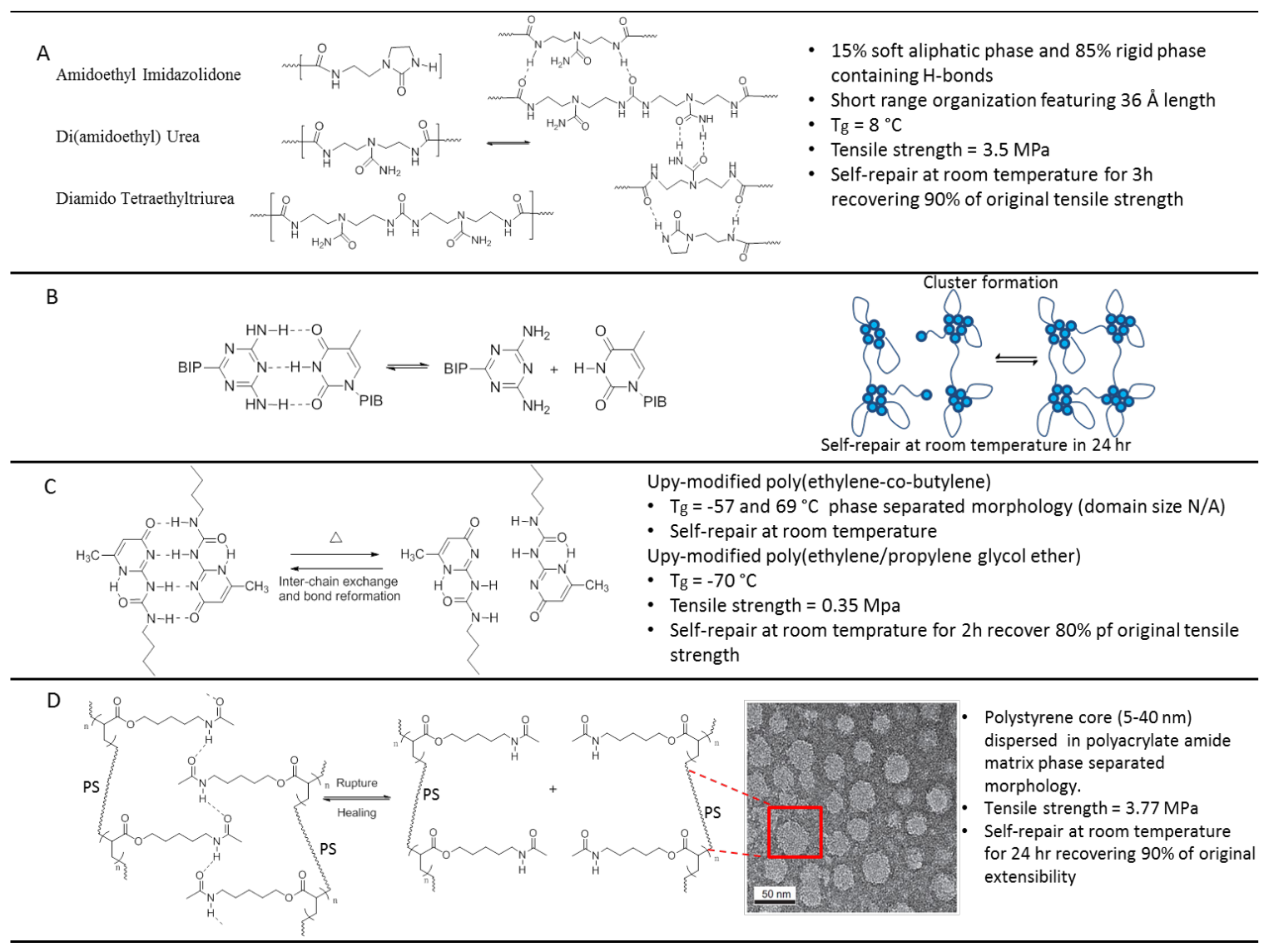

Figure 7. 


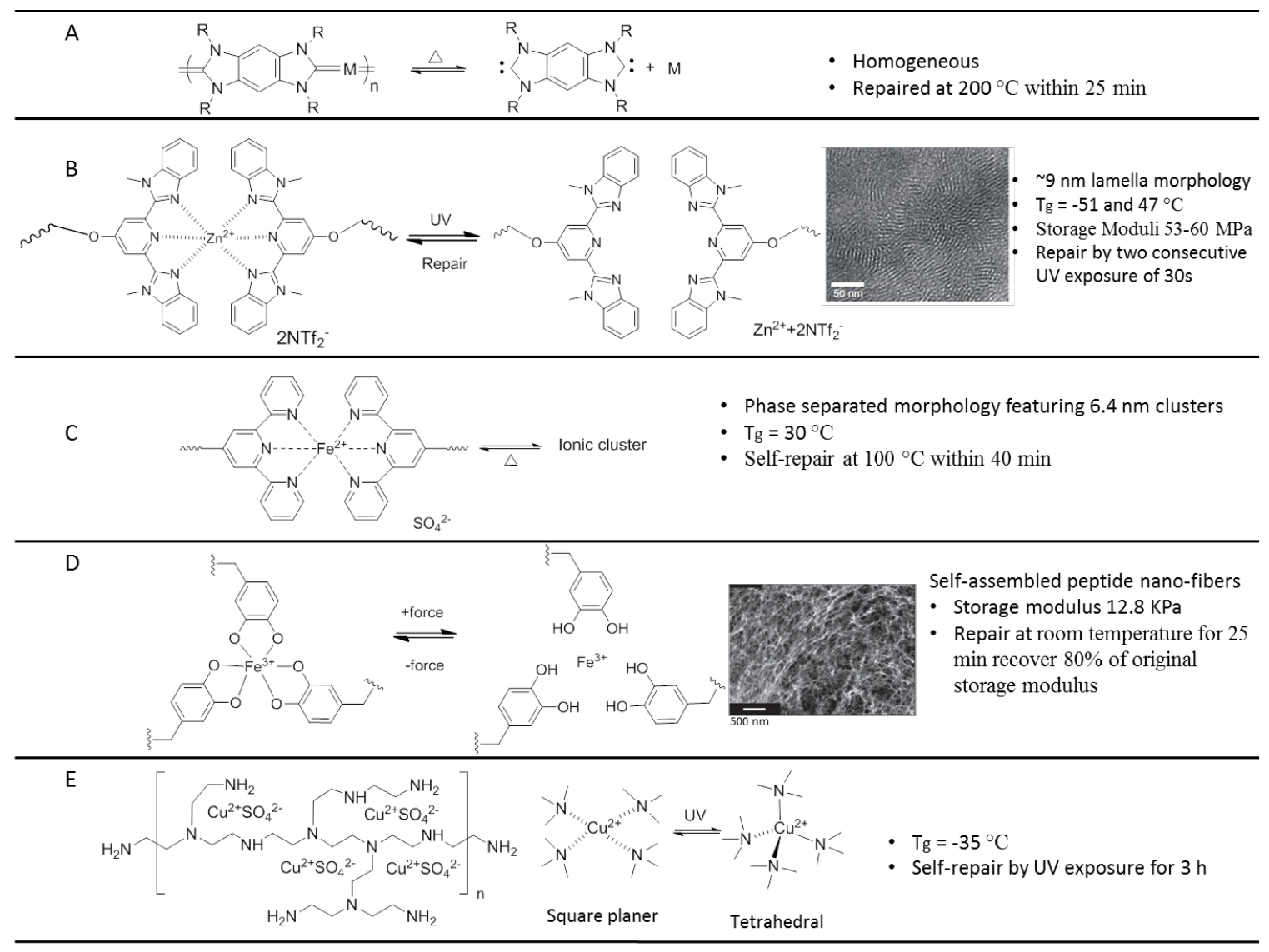

Figure 8.. Reproduced with permission from the Royal Society of Chemistry. 

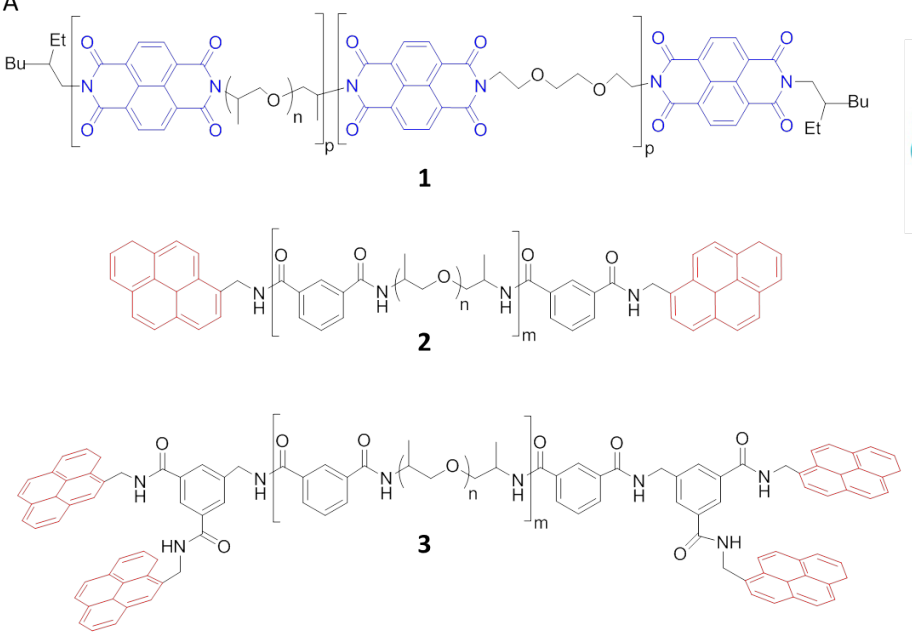

C

\begin{tabular}{ccccc}
\hline Blend & $\begin{array}{c}\text { Tensile } \\
\text { modulus } \\
(\mathrm{Mpa})\end{array}$ & $\begin{array}{c}\text { Tensile } \\
\text { strength } \\
(\mathrm{Mpa})\end{array}$ & $\begin{array}{c}\text { Elongation } \\
\text { at break }(\%)\end{array}$ & $\begin{array}{c}\text { Self-healing conditions } \\
(100 \% \text { recovery of } \\
\text { tensile modulus })\end{array}$ \\
\hline$[1+2]$ & $1.2 \pm 0.2$ & $2.7 \pm 0.7$ & $9.3 \pm 1.5$ & $140^{\circ} \mathrm{C}$ for $160 \mathrm{~min}$ \\
{$[1+3]$} & $1.8 \pm 0.4$ & $6.7 \pm 1.2$ & $53 \pm 7$ & $87^{\circ} \mathrm{C}$ for 5 min
\end{tabular}
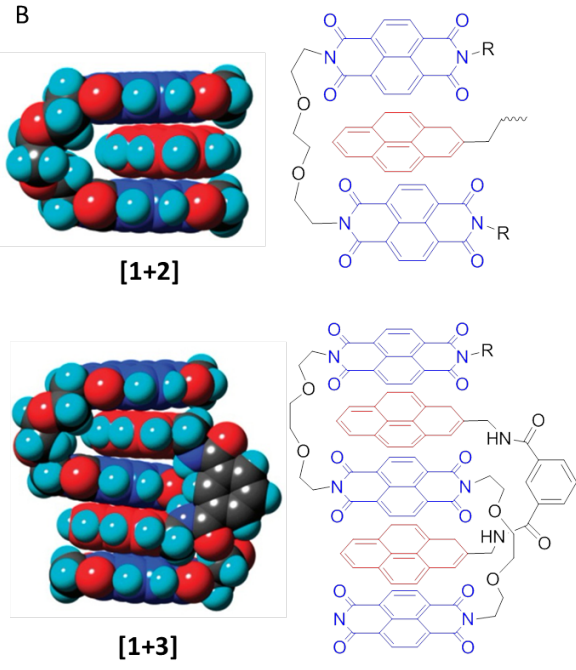

Figure 9. 
A

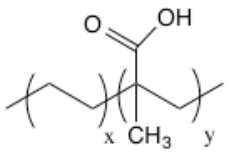

Poly(ethylene-co-methacrylic acid) (EMAA)
B

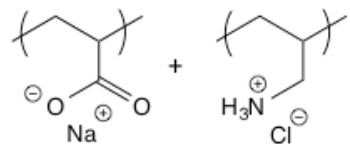

Poly(acrylic acid) (PAA)/poly(allylamine hydrochloride) (PAH) compact polyelectrolyte complexes (CoPECs)
C<smiles>CC(C)(C)CC(C[NH3+])C(C)(C)Cl</smiles>

Poly(allylamine hydrochloride) (PAH)/ tripolyphosphate (TPP) ionic gel

\section{Figure 10.}



A
B
C
D

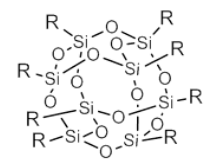

Surface Reactive Groups

Matrix Reactive Groups

Surface-Matrix

Covalent Bonding

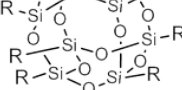

$-\mathrm{COOH}$

$\longrightarrow \quad m-\mathrm{H}_{\mathrm{N}} \mathrm{O}-\mathrm{C}-m$

Silica NanoCage

$-\mathrm{COCl}$

$-\mathrm{NH}_{2}$

III-H $-\mathrm{H}$

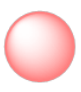

Nanoparticle

$-\mathrm{OH}+$

$\mathrm{RO}-\mathrm{Si}-\mathrm{or}-\mathrm{NCO} \longrightarrow m-\mathrm{O}-\mathrm{Si}-m$ or $m-\mathrm{O}-\mathrm{O}-\mathrm{C}-\mathrm{N}-m$
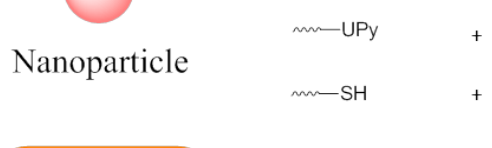

UPy $=m$

$\longrightarrow \quad$ Reversible Bonding

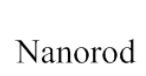

$+$

HS - m

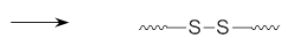

Nanorod

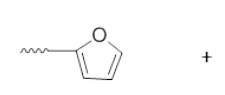

$\left.\right|_{0} ^{N-m}$
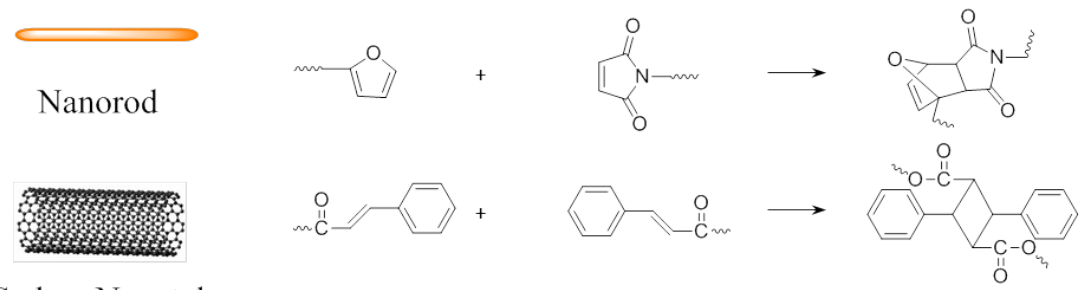

Carbon Nanotube
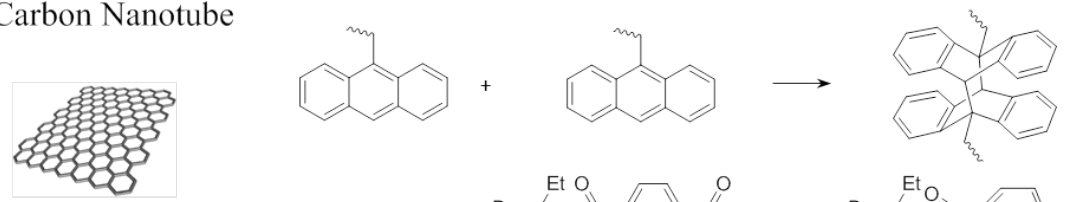

Graphene

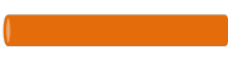

Nano Fiber
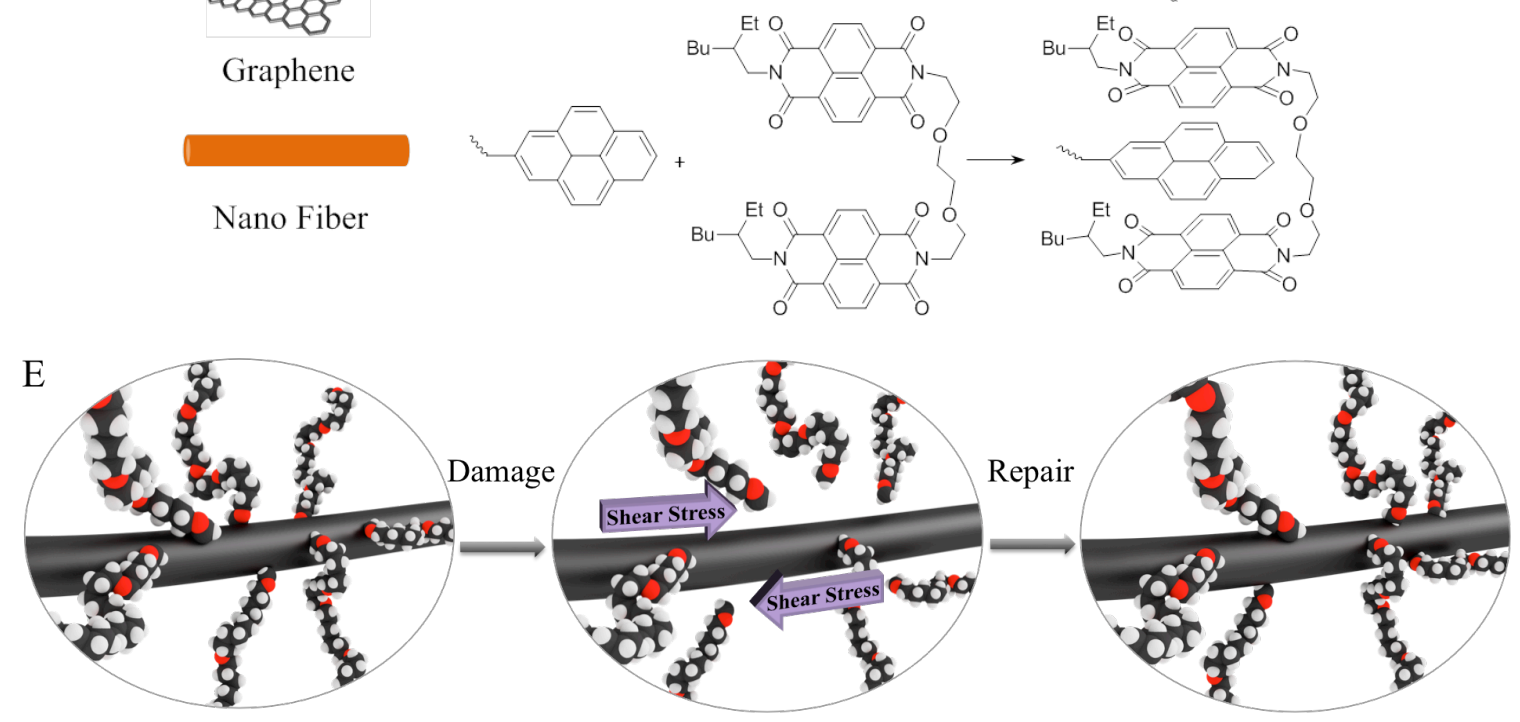

Figure 11. 
A
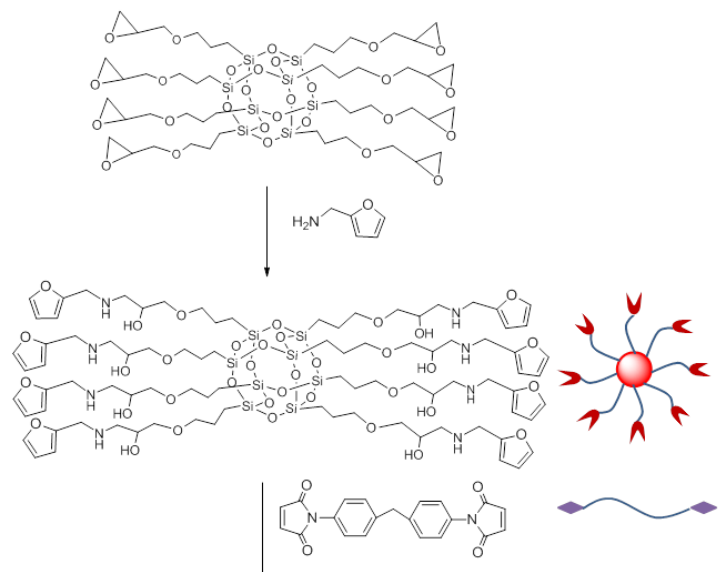

《ID+
B

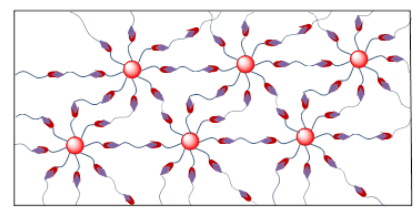

Damage Repair

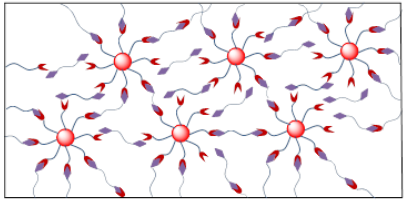

Figure 12. 


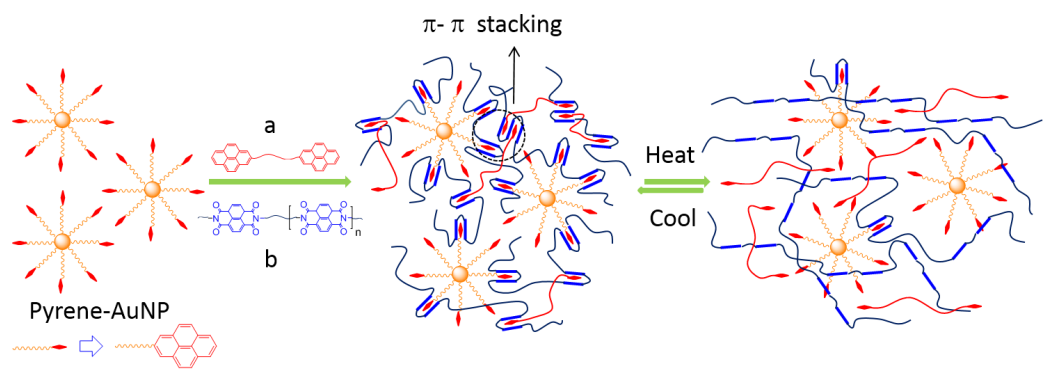

Figure 13. 


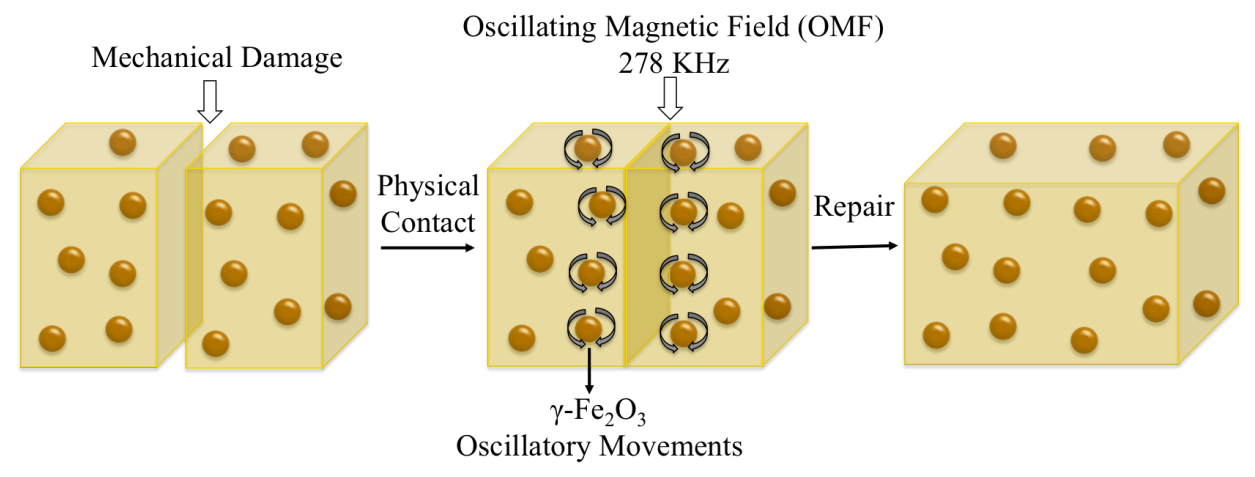

Figure 14. 
A

B
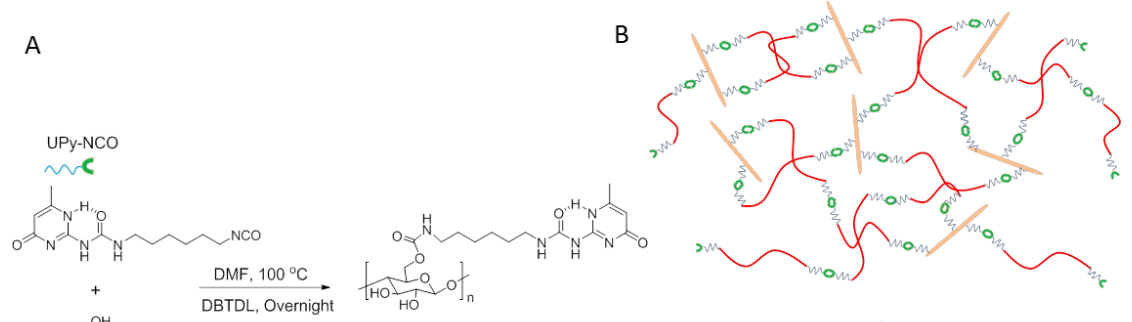
$\frac{\mathrm{HONO}_{\mathrm{HO}} \underbrace{\mathrm{OH}}_{\mathrm{CN}}]_{\mathrm{n}}}{\mathrm{C}_{\mathrm{O}}}$

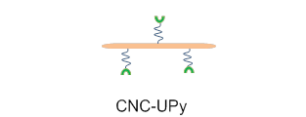

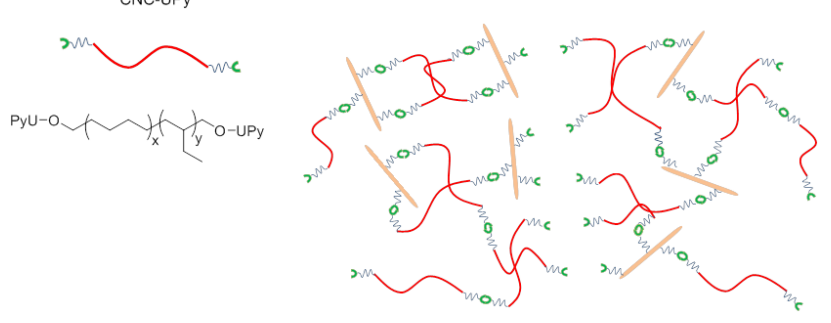

Figure 15. 
A

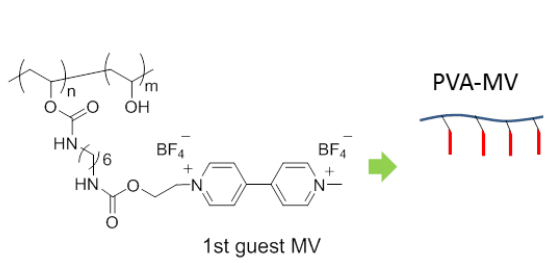

B

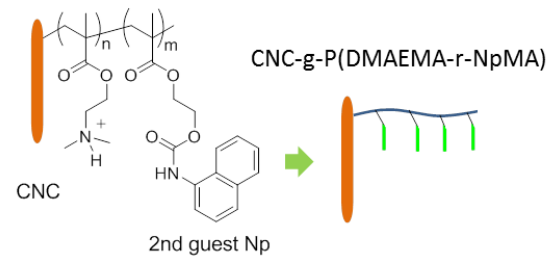

C

D Host-guest crosslinking by three-component recognition

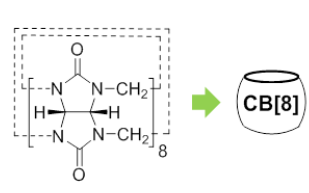

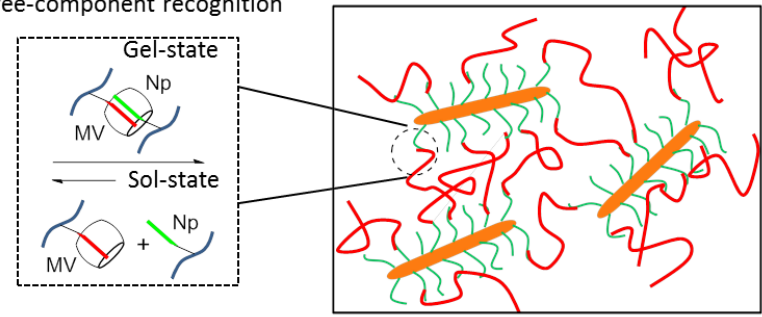

Figure 16. 


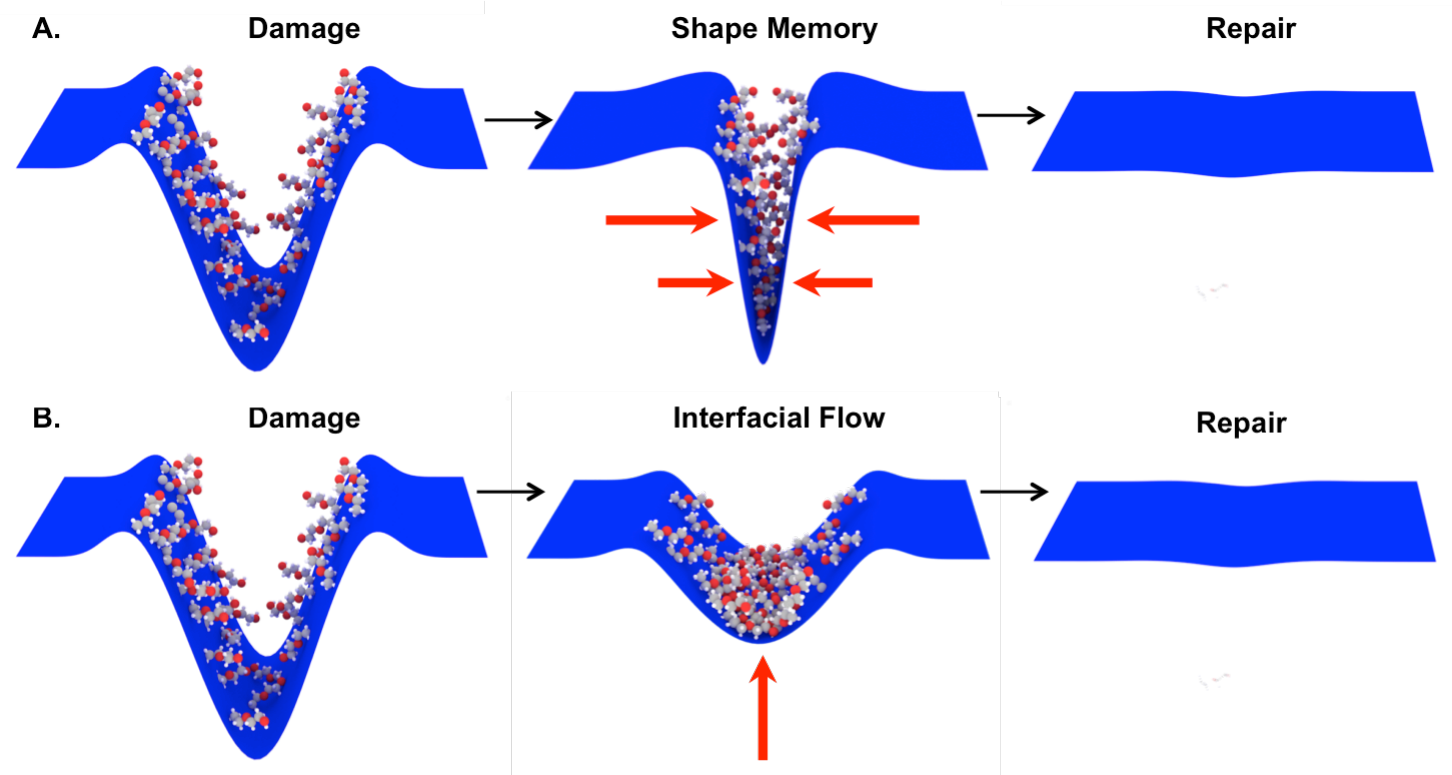

Figure 17. 
Table 1. Cycloaddition reactions responsible for self-repair. The corresponding physical properties and self-repair conditions are listed for corresponding polymer matrices.

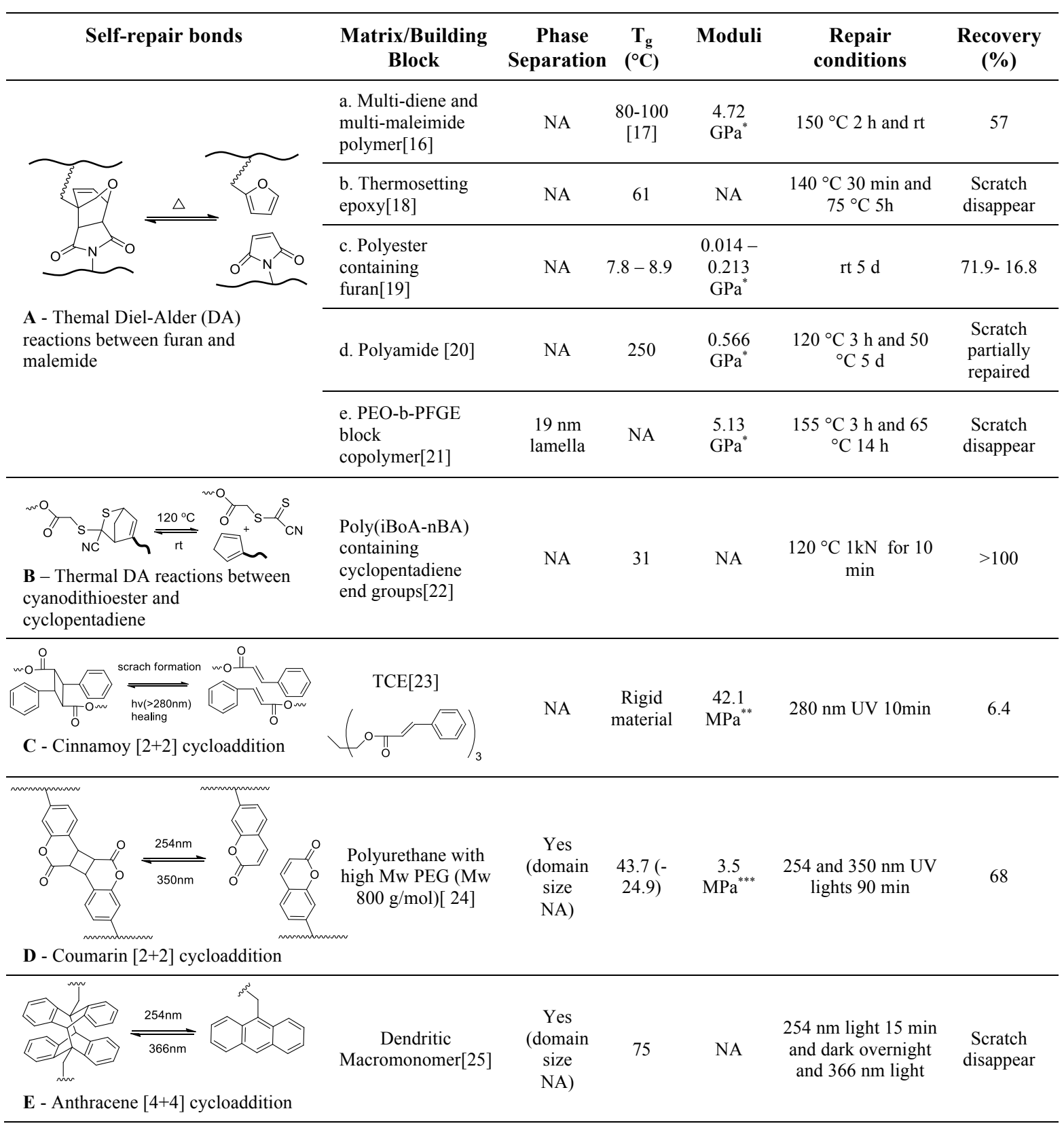

*:Yong's modulus; **: flexural strength; ***: tensile strength, $\mathrm{rt}$ - room temperature. 
Table 2. Physical and self-repairing properties of S-S based homo- and heterogeneous networks.

\begin{tabular}{|c|c|c|c|c|c|c|}
\hline $\begin{array}{l}\text { Self- } \\
\text { repairing } \\
\text { bond }\end{array}$ & Matrix & $\begin{array}{c}\text { Phase } \\
\text { heterogeneity }\end{array}$ & $\mathbf{T}_{\mathrm{g}}\left({ }^{\circ} \mathrm{C}\right)$ & $\begin{array}{l}\text { Strength at } \\
\text { break }\end{array}$ & $\begin{array}{c}\text { Repair } \\
\text { conditions }\end{array}$ & $\begin{array}{c}\text { Recovery } \\
(\%)\end{array}$ \\
\hline \multirow{4}{*}{ S-S } & A. Aliphatic epoxy resin[32] & No & -35 & $1.3 \mathrm{MPa}$ & $60^{\circ} \mathrm{C} 1 \mathrm{~h}$ & 96.9 \\
\hline & $\begin{array}{l}\text { B. Poly (n-butyl acryalte) } \\
\text { grafted poly (ethylene glycol } \\
\text { diacylate) star polymers[33] }\end{array}$ & Yes (size NA) & NA & $20 \mathrm{kPa}$ & rt $20 \mathrm{~min}-24 \mathrm{~h}$ & NA \\
\hline & $\begin{array}{l}\text { C. Epoxy enhanced with } \\
\text { aromatic segments[34] }\end{array}$ & NA & -3.6 & $\begin{array}{l}0.18-0.52 \\
\mathrm{MPa}\end{array}$ & $65^{\circ} \mathrm{C} 240 \mathrm{~min}$ & 100 \\
\hline & $\begin{array}{l}\text { D. Epoxy enhanced with } \\
\text { alkoxide inorganic phase[35] }\end{array}$ & Yes (size NA) & $-11--9$ & $1.6 \mathrm{MPa}$ & $60-70{ }^{\circ} \mathrm{C} 10 \mathrm{~min}$ & 100 \\
\hline
\end{tabular}


Table 3. Self-repair and physical properties of alkoxyamine crosslinked polystyrenes in different monomer ratios.

\begin{tabular}{|c|c|c|c|c|c|}
\hline Self-repair bonds & Matrix & $\mathrm{T}_{\mathrm{g}}\left({ }^{\circ} \mathrm{C}\right)$ & $\begin{array}{c}\text { Phase } \\
\text { heterogeneity }\end{array}$ & $\begin{array}{l}\text { Repair } \\
\text { conditions }\end{array}$ & $\begin{array}{l}\text { Recovery } \\
(\%)\end{array}$ \\
\hline \multirow{3}{*}{ Alkoxyamine[42] } & $\begin{array}{l}\text { Polystyrene: alkoxyamine } \\
=5: 1\end{array}$ & 125 & Not detected & & 26.4 \\
\hline & $\begin{array}{c}\text { Polystyrene: alkoxyamine } \\
=7.5: 1\end{array}$ & (108) 125 & $\begin{array}{l}\text { Yes (sizes } \\
\text { N/A) }\end{array}$ & $\begin{array}{l}130^{\circ} \mathrm{C} \\
2.5 \mathrm{hr}\end{array}$ & 75.7 \\
\hline & $\begin{array}{l}\text { Polystyrene: alkoxyamine } \\
=10: 1\end{array}$ & 125 & Not detected & & 32.6 \\
\hline
\end{tabular}

